\title{
Linear and nonlinear eigenvalue problems for Dirac systems in unbounded domains
}

\author{
Anna Capietto, Walter Dambrosio and Duccio Papini
}

\begin{abstract}
We first study the linear eigenvalue problem for a planar Dirac system in the open half-line and describe the nodal properties of its solutions by means of the rotation number. We then give a global bifurcation result for a planar nonlinear Dirac system in the open half-line. As an application, we provide a global continuum of solutions of the nonlinear Dirac equation which have a special form.
\end{abstract}

Mathematics Subject Classification (2000). 34C23, 34B09, 34L40.

Keywords. Dirac system, Eigenvalue problem, Rotation number, Global bifurcation.

\section{Introduction}

In this paper we give a global bifurcation result (Theorem 4.3) for a nonlinear Dirac system in $\mathbb{R}^{2}$ of the form

$$
J z^{\prime}+P(x) z=\lambda z+S(x, z) z, \quad x>0, \quad \lambda \in \mathbb{R}, \quad z=(u, v) \in \mathbb{R}^{2},
$$

where

$$
J=\left(\begin{array}{ll}
0 & 1 \\
-1 & 0
\end{array}\right)
$$

and $P(x), S(x, z)$ are continuous symmetric matrices, for every $x>0$ and $z \in \mathbb{R}^{2}$. We will be interested in solutions $z$ of (1.1) belonging to the space

$$
D_{0}=\left\{z \in L^{2}(0,+\infty): z \in A C(0,+\infty), J z^{\prime}+P(\cdot) z \in L^{2}(0,+\infty)\right\} .
$$

Under the auspices of GNAMPA-I.N.d.A.M., Italy. The work of the first two authors has been performed in the frame of the M.I.U.R. Project 'Topological and Variational Methods in the Study of Nonlinear Phenomena'; the work of the third author has been performed in the frame of the M.I.U.R. Project 'Nonlinear Control: Geometrical Methods and Applications' and of the GNAMPA-I.N.d.A.M. project "Equazione di evoluzione degeneri e singolari: controllo e applicazioni". 
In particular, the solutions are convergent to zero at zero and at infinity. This choice is strictly related to the spectral properties of the linear operator $\tau z=J z^{\prime}+P(x) z$ and to the possibility of considering self-adjoint extensions of $\tau$ (see Sect. 3).

When $P$ has the form

$$
P(x)=P_{V, k, \mu_{a}}(x)=\left(\begin{array}{ll}
-1+V(x) & -\frac{k}{x}-\mu_{a} V^{\prime}(x) \\
-\frac{k}{x}-\mu_{a} V^{\prime}(x) & 1+V(x)
\end{array}\right), \quad x>0,
$$

the differential operator $z \mapsto J z^{\prime}+P(\cdot) z$ coincides with the radially symmetric Dirac operator with or without anomalous magnetic moment (cf. [15, 18,23,24] and Sect. 4.2). In this context $V \in C^{1}(0,+\infty)$ represents an electrostatic potential, $\mu_{a} \in \mathbb{R}$ an anomalous magnetic moment and $k \in \mathbb{Z} \backslash\{0\}$ (see [23]). For a comprehensive treatment of linear and nonlinear Dirac systems, we refer to the paper by Esteban [14]. As it is explained in detail in Sect. 4.2, nonlinear systems of the form (1.1) arise, for some $S$, when one is interested in solutions of a nonlinear Dirac PDE which have a special form (cf. (4.49)).

The study of global bifurcation problems for second order equations in unbounded intervals was initiated in the 1970s by Stuart [22] and Dancer $[7,8]$. More recent results have been given by Rabier and Stuart [16], Secchi and Stuart [20], the first and second author [4] and the authors [5].

In [4] it is considered the particular case when the r.h.s. of (1.1) (and the function $S$ ) is regular at zero. We are now able to avoid this restriction and, as a consequence, to treat the physically relevant Dirac operator.

Having in mind a bifurcation result, a comprehensive knowledge of the linear eigenvalue problem

$$
J z^{\prime}+P(x) z=\lambda z, \quad x>0, \quad \lambda \in \mathbb{R}, \quad z=(u, v) \in \mathbb{R}^{2}
$$

is necessary. More precisely, we have to study the existence of eigenvalues and their "nodal properties". To this end, in Subsection 2.1, assuming $\left(\mathcal{P}_{1}\right),\left(\mathcal{P}_{2}\right)$, $\left(\mathcal{P}_{3}\right)$ for the matrix $P$, we first describe (Lemmas 2.6 and 2.13) the behaviour of the solutions of the linear system (1.3) when $x \rightarrow+\infty$ and $x \rightarrow 0^{+}$. As in [4], we apply the Levinson theorem [13] on the asymptotic properties of solutions of linear equations and, by means of a suitable change of variables, we manage to treat the singularity at zero as well. Using the results of Subsection 2.1, we develop in Subsection 2.2 an oscillatory theory for nontrivial solutions of (2.1) based on the study of the asymptotic behaviour of the angular coordinate $\theta$ in the phase-plane (cf. the book by Weidmann [24]). It is interesting to observe that, contrary to the case of second order equations, in case of planar Diractype systems the angular coordinate is not, in general, an increasing function of $x$. However, we are able to guarantee (Propositions 2.16 and 2.17) that the limits

$$
\theta(+\infty, \lambda)=\lim _{x \rightarrow+\infty} \theta(x, \lambda), \quad \theta(0)=\lim _{x \rightarrow 0^{+}} \theta(x, \lambda)
$$

exist and are finite. We can thus give the definition of

$$
\operatorname{rot}(z)=\frac{\theta(+\infty, \lambda)-\theta(0)}{\pi}
$$


the rotation number of a solution $z$ to (1.3). Roughly speaking, the unboundedness of the interval and the singularity at zero do not prevent solutions to perform only a finite number of rotations around the origin (as in the regular case). A nontrivial phase-plane analysis leads then to some useful continuity properties of the angular function near zero and infinity (Propositions 2.21 and 2.22).

In Sect. 3 we study the spectral theory for the linear operator formally defined by

$$
\tau z=J z^{\prime}+P(x) z, \quad x>0 .
$$

More precisely, standard arguments from [24] ensure that $\tau$ is in the limit point case at infinity and at zero and that there exists a unique self-adjoint realization $A_{0}$ (cf. (3.2)) of $\tau$ having (when $P$ has the form (1.2)) essential spectrum $\sigma_{\text {ess }}\left(A_{0}\right)=(-\infty,-1] \cup[1,+\infty)$. Then, the (nontrivial) question of characterizing eigenvalues of $A_{0}$ is tackled by the results of Subsection 2.2. Finally, we give results on the existence and accumulation of eigenvalues of $A_{0}$ at the boundary of the interval $(-1,1)$ which are based on the oscillatory behaviour of the solutions for a value of $\lambda$ corresponding to one of the extrema of the essential spectrum; similar results can be found in the case of secondorder differential operators in the book by Dunford and Schwartz [12] and in case of Dirac operators (without any knowledge of the nodal properties of the corresponding eigenvalues) in the paper by Schmid and Tretter [18].

Taking advantage of all the results described above, in Subsection 4.1 we give a global bifurcation result (Theorem 4.3) for system (1.1). Due to the fact that we are dealing with an unbounded interval, we face a lack of compactness; this difficulty is overcome by applying an abstract bifurcation result due to Stuart [22]. A more precise description of the continuum emanating from eigenvalues of odd multiplicity of the linear operator $\tau$ is then performed (as we did in [4]) in Theorem 4.8; to this aim, we develop a continuity-connectivity argument based on a linearization approach and on the properties of the rotation number of a solution to (1.1) (cf. (4.5), (4.33) and Proposition 4.7).

Finally, in Subsection 4.2 we consider the partial differential equation

$$
\begin{gathered}
i \sum_{j=1}^{3} \alpha_{j} \frac{\partial \psi}{\partial x_{j}}-\beta \psi-V(\|x\|) \psi+i a \sum_{j=1}^{3} \alpha_{j} \frac{\partial V(\|x\|)}{\partial x_{j}} \psi \\
\quad=\lambda \psi+\gamma(\|x\|) F(\langle\beta \psi, \psi\rangle) \beta \psi, \quad x \in \mathbb{R}^{3}, a \in \mathbb{R},
\end{gathered}
$$

where $\psi: \mathbb{R}^{3} \rightarrow \mathbb{C}^{4}, V \in C((0,+\infty), \mathbb{R})$ and $\gamma \in C((0,+\infty), \mathbb{R})$ satisfy suitable assumptions, $\langle\cdot, \cdot\rangle$ denotes the scalar product in $\mathbb{C}^{4}$ and $\alpha_{j}(j=1,2,3)$ and $\beta$ are the $4 \times 4$ Dirac matrices (see Subsection 4.2). Set

$$
H_{0} \psi=i \sum_{j=1}^{3} \alpha_{j} \frac{\partial \psi}{\partial x_{j}}-\beta \psi, \quad \forall \psi \in H_{0}^{1}\left(\mathbb{R}^{3}\right) \subset L^{2}\left(\mathbb{R}^{3}\right) .
$$

It is well-known (cf. the book by Thaller [23]), that there exist suitable subspaces of $L^{2}\left(S^{2}\right)$ s.t. the restriction of the linear operator $H_{0}-V+i a \alpha \cdot \nabla V$ to each of these subspaces can be represented by an ordinary differential operator of the form $\tau$. A remark on the physical meaning of the partial wave 
subspaces can be found in Remark 4.10 in Sect. 4.2. It is interesting to observe (on the lines of a paper by Cacciafesta [3]) that there are nonlinear terms $F(\langle\beta \psi, \psi\rangle) \beta \psi$ in (1.7) which leave the above described subspaces invariant. These appear, among others, in the so-called Soler model and are the most interesting from a physical point of view (cf. $[17,21]$ ). On the same lines, we refer also to the contributions by Balabane et al. [2], Ding and Ruf [10], Ding et al. [9], Dong and Xie [11] and references therein.

Our contribution (Theorem 4.11) provides the existence of a global continuum of solutions of the nonlinear PDE (1.7) which have a special form (i.e. which belong to one of the above mentioned subspaces). To the authors' knowledge, Theorem 4.11 is the first global bifurcation result for a nonlinear Dirac-type equation of the form (1.7). In the particular case $V \equiv 0$, Balabane et al. [1] gave a multiplicity result for solutions (having prescribed nodal properties) to a system of ODEs of the form (4.51). For multiplicity results via critical point theory for the nonlinear Dirac PDE, we refer to Theorem 3.3 in [14] (in case $V \equiv 0$ ) and to the paper by Ding and Ruf [10] (for a potential that includes the Coulomb case). On the other hand, in the particular case of linear Dirac-type systems of ODEs, Schmid and Tretter [18] have given results for the eigenvalue problem for some special choice of the potential $V$.

In what follows, we will denote by $M_{S}^{2}$ the set of symmetric $2 \times 2$ matrices.

\section{Linear Dirac systems}

In this Section we consider a linear system of the form

$$
J z^{\prime}+P(x) z=\lambda z, \quad x>0, \quad \lambda \in \mathbb{R}, \quad z=(u, v) \in \mathbb{R}^{2} ;
$$

by a solution of $(2.1)$ we mean a function $z \in A C_{\text {loc }}(0,+\infty)$ satisfying (2.1) almost everywhere in $(0,+\infty)$. In the next sections we will be interested in solutions $z \in L^{2}(0,+\infty)$ or $z \in H^{1}(0,+\infty)$; hence, in describing the solutions of $(2.1)$ we will point out, when possible, if they belong to $L^{2}(0,+\infty)$ or to $H^{1}(0,+\infty)$.

We assume that $P \in C\left((0,+\infty), M_{S}^{2}\right)$ and we denote by $p_{i j}$ its coefficients, as usual. For each pair of real numbers $\mu_{-}<\mu^{+}$, let us consider the class $\mathcal{P}_{\mu}$ of continuous maps $P:(0,+\infty) \longrightarrow M_{S}^{2,2}$ satisfying the following conditions:

$\left(\mathcal{P}_{1}\right)$ There exists $q_{\infty} \geq 1$ such that

$$
\lim _{x \rightarrow+\infty} P(x)=\left(\begin{array}{cc}
\mu^{-} & 0 \\
0 & \mu^{+}
\end{array}\right)=: P_{\infty}
$$

and

$$
\int_{1}^{+\infty}\left\|R_{\infty}(x)\right\|^{q_{\infty}} d x<+\infty,
$$

where $R_{\infty}(x)=P(x)-P_{\infty}$, for every $x \geq 1$. 
$\left(\mathcal{P}_{2}\right)$ There exist $\beta \geq 1, P^{*} \in M_{S}^{2}$ and $q_{0} \geq 1$ such that

$$
\lim _{x \rightarrow 0^{+}} x^{\beta} P(x)=P^{*}
$$

and

$$
\int_{0}^{1} \frac{1}{x^{\beta}}\left\|R_{0}(x)\right\|^{q_{0}} d x<+\infty,
$$

where $R_{0}(x)=x^{\beta} P(x)-P^{*}$, for every $x \in(0,1)$.

$\left(\mathcal{P}_{3}\right)$ The matrix $P^{*}$ satisfies

$$
\begin{array}{r}
\operatorname{det} P^{*}<-1 / 4 \quad \text { if } \beta=1 \\
\operatorname{det} P^{*}<0 \text { if } \beta>1 .
\end{array}
$$

In what follows, we write $\Lambda=\left(\mu^{-}, \mu^{+}\right)$.

Remark 2.1. 1. We observe that assumption $\left(\mathcal{P}_{2}\right)$ implies that $(2.1)$ has a singularity for $x \rightarrow 0^{+}$; indeed, from (2.4) and the fact that $P^{*}$ is not the zero-matrix (since its determinant is negative in any case), we deduce that

$$
p_{i j}(x) \sim \frac{p_{i j}^{*}}{x^{\beta}}, \quad x \rightarrow 0^{+} \quad(i, j=1,2)
$$

and, in particular, that $p_{i j} \notin L^{1}(0,1)$.

(2) Let us also observe that, for a particular choice of $P$, the differential operator given in (2.1) coincides with the radially symmetric Dirac operator with or without anomalous magnetic moment (cf. [15, 18, 23,24] and Sect. 4.2); indeed, this is the situation when $P$ has the form

$$
P(x)=P_{V, k, \mu_{a}}(x)=\left(\begin{array}{cc}
-1+V(x) & -\frac{k}{x}-\mu_{a} V^{\prime}(x) \\
-\frac{k}{x}-\mu_{a} V^{\prime}(x) & 1+V(x)
\end{array}\right), \quad x>0,
$$

where $V \in C^{1}(0,+\infty)$ is an electrostatic potential, $\mu_{a} \in \mathbb{R}$ is an anomalous magnetic moment and $k \in \mathbb{Z} \backslash\{0\}$ (see [23]).

Let us assume that $V$ satisfies the following conditions:

$$
\begin{aligned}
& V(x)=\frac{\gamma_{\infty}}{x_{\infty}}+R_{V, \infty}(x), \alpha_{\infty}>0, \\
& x^{\alpha_{\infty}} R_{V, \infty}=o(1), \quad x^{\alpha_{\infty}+1} R_{V, \infty}^{\prime}=o(1), x \rightarrow+\infty
\end{aligned}
$$

and

$$
V(x)=\frac{\gamma_{0}}{x^{\alpha_{0}}}+R_{V, 0}(x), \quad \alpha_{0}>0,
$$

where

$$
\text { if } \mu_{a}=0:\left\{\begin{array}{l}
\alpha_{0}=1, \quad x R_{V, 0}=o(1), \quad x \rightarrow 0^{+}, \\
\int_{0}^{1} \frac{1}{x}\left|x R_{V, 0}(x)\right|^{q^{\prime}} d x<+\infty, \quad q^{\prime} \geq 1, \\
\gamma_{0}^{2}<k^{2}-1 / 4
\end{array}\right.
$$


and

$$
\text { if } \mu_{a} \neq 0:\left\{\begin{array}{l}
x^{\alpha_{0}} R_{V, 0}=o(1), \quad x^{\alpha_{0}+1} R_{V, 0}^{\prime}=o(1), \quad x \rightarrow 0^{+}, \\
\int_{0}^{1} \frac{1}{x^{\alpha_{0}+1}}\left|x^{\alpha_{0}+1} R_{V, 0}^{\prime}(x)\right|^{q^{\prime \prime}} d x<+\infty, \quad q^{\prime \prime} \geq 1, \\
\gamma_{0} \neq 0 .
\end{array}\right.
$$

Under these conditions, assumption $\left(\mathcal{P}_{1}\right)$ is satisfied with $\mu^{ \pm}= \pm 1$; indeed, we obviously have

$$
\lim _{x \rightarrow+\infty} P_{V, k, \mu_{a}}(x)=\lim _{x \rightarrow+\infty}\left(\begin{array}{cc}
-1+V(x) & -\frac{k}{x}-\mu_{a} V^{\prime}(x) \\
-\frac{k}{x}-\mu_{a} V^{\prime}(x) & 1+V(x)
\end{array}\right)=\left(\begin{array}{cc}
-1 & 0 \\
0 & 1
\end{array}\right) .
$$

Moreover, the matrix $R_{\infty}$ in $(2.3)$ is given by

$$
R_{\infty}(x)=\left(\begin{array}{cc}
V(x) & -\frac{k}{x}-\mu_{a} V^{\prime}(x) \\
-\frac{k}{x}-\mu_{a} V^{\prime}(x) & V(x)
\end{array}\right), \quad \forall x>0 ;
$$

from (2.9) we deduce that $V, R_{V, \infty} \in L^{q}(1,+\infty)$, for every $q>1 / \alpha_{\infty}$, and $R_{V, \infty}^{\prime} \in L^{p}(1,+\infty)$, for every $p>1 /\left(\alpha_{\infty}+1\right)$, while we plainly have $k / x \in$ $L^{s}(1,+\infty)$, for every $s>1$. By observing that all the functions $V, R_{V, \infty}, R_{V, \infty}^{\prime}$, $k / x$ go to zero at infinity, we conclude that (2.3) is satisfied with $q_{\infty}=1 /\left(\alpha_{\infty}+\right.$ 1).

As far as $\left(\mathcal{P}_{2}\right)$ and $\left(\mathcal{P}_{3}\right)$ are concerned, a crucial role is played by the constants $\gamma_{0}$ and $\mu_{a}$, as it is evident from the assumptions on $V$. Indeed, let us first discuss the case $\mu_{a}=0$; in this situation, taking $\alpha_{0}=1$ and $\beta=\alpha_{0}=1$ in (2.4), we have

$$
\begin{aligned}
P^{*} & =\lim _{x \rightarrow 0^{+}} x P_{V, k, 0}(x) \\
& =\lim _{x \rightarrow 0^{+}}\left(\begin{array}{cc}
-x+x V(x) & -k \\
-k & x+x V(x)
\end{array}\right)=\left(\begin{array}{cc}
\gamma_{0} & -k \\
-k & \gamma_{0}
\end{array}\right)
\end{aligned}
$$

and

$$
R_{0}(x)=x P(x)-P^{*}=\left(\begin{array}{ll}
-x+x V(x)-\gamma_{0} & 0 \\
0 & x+x V(x)-\gamma_{0}
\end{array}\right) .
$$

Hence, from (2.10) and (2.11) we infer

$$
\int_{0}^{1} \frac{1}{x} \| R_{0}(x)||^{q^{\prime}} d x \leq 2^{q^{\prime}-1} \int_{0}^{1}\left(x^{q^{\prime}-1}+x^{q^{\prime}-1}\left|R_{V, 0}(x)\right|^{q^{\prime}}\right) d x<+\infty,
$$

concluding that (2.5) holds true with $q_{0}=q^{\prime}$. Moreover, the last relation in (2.11) guarantees that (2.6) is fulfilled.

Suppose now $\mu_{a} \neq 0$; taking $\beta=\alpha_{0}+1$ in (2.4), we have

$$
\begin{aligned}
P^{*} & =\lim _{x \rightarrow 0^{+}} x^{\alpha_{0}+1} P_{V, k, \mu_{a}}(x)= \\
& =\lim _{x \rightarrow 0^{+}}\left(\begin{array}{cc}
-x^{\alpha_{0}+1}+x^{\alpha_{0}+1} V(x) & -k x^{\alpha_{0}}-\mu_{a} x^{\alpha_{0}+1} V^{\prime}(x) \\
-k x^{\alpha_{0}}-\mu_{a} x^{\alpha_{0}+1} V^{\prime}(x) & x^{\alpha_{0}+1}+x^{\alpha_{0}+1} V(x)
\end{array}\right) \\
& =\left(\begin{array}{ll}
0 & \mu_{a} \alpha_{0} \gamma_{0} \\
\mu_{a} \alpha_{0} \gamma_{0} & 0
\end{array}\right)
\end{aligned}
$$


and

$$
\begin{aligned}
& R_{0}(x)=x^{\alpha_{0}+1} P(x)-P^{*} \\
& \quad=\left(\begin{array}{ll}
-x^{\alpha_{0}+1}+x^{\alpha_{0}+1} V(x) & -k x^{\alpha_{0}}-\mu_{a} x^{\alpha_{0}+1} V^{\prime}(x)-\mu_{a} \alpha_{0} \gamma_{0} \\
-k x^{\alpha_{0}}-\mu_{a} x^{\alpha_{0}+1} V^{\prime}(x)-\mu_{a} \alpha_{0} \gamma_{0} & x^{\alpha_{0}+1}+x^{\alpha_{0}+1} V(x)
\end{array}\right) .
\end{aligned}
$$

Now, let $q_{0}>\max \left(q^{\prime \prime}, \alpha_{0}\right)$; from (2.10) and (2.12) we infer

$$
\begin{aligned}
& \int_{0}^{1} \frac{1}{x^{\alpha_{0}+1}}\left|x^{\alpha_{0}+1}\right|^{q_{0}} d x=\int_{0}^{1} \frac{1}{x^{\left(\alpha_{0}+1\right)\left(1-q_{0}\right)}} d x<+\infty \\
& \int_{0}^{1} \frac{1}{x^{\alpha_{0}+1}}\left|x^{\alpha_{0}+1} V(x)\right|^{q_{0}} d x \leq 2^{q_{0}-1} \int_{0}^{1} \frac{x^{q_{0}}}{x^{\alpha_{0}+1}}\left(\gamma_{0}^{q_{0}}+\left|x^{\alpha_{0}} R_{V, 0}(x)\right|^{q_{0}}\right) d x<+\infty \\
& \int_{0}^{1} \frac{1}{x^{\alpha_{0}+1}}\left|k x^{\alpha_{0}}\right|^{q_{0}} d x=|k|^{q_{0}} \int_{0}^{1} \frac{1}{x^{1+\left(1-q_{0}\right) \alpha_{0}}} d x<+\infty \\
& \int_{0}^{1} \frac{1}{x^{\alpha_{0}+1}}\left|-\mu_{a} x^{\alpha_{0}+1} V^{\prime}(x)-\mu_{a} \alpha_{0} \gamma_{0}\right|^{q_{0}} d x \\
& =\left|\mu_{a}\right|^{q_{0}} \int_{0}^{1} \frac{1}{x^{\alpha_{0}+1}}\left|x^{\alpha_{0}+1} R_{V, 0}^{\prime}(x)\right|^{q^{\prime \prime}}\left|x^{\alpha_{0}+1} R_{V, 0}^{\prime}(x)\right|^{q_{0}-q^{\prime \prime}} d x<+\infty,
\end{aligned}
$$

concluding again that (2.5) holds true. Moreover, the last relation in (2.12) guarantees that $(2.7)$ is fulfilled.

The fact that $P \in \mathcal{P}_{\mu}$, and in particular the fact that $P$ satisfies $\left(\mathcal{P}_{3}\right)$, is related to the spectral properties of the operator $\tau: z \rightarrow J z^{\prime}+P(x) z$; indeed, as we will see at the beginning of Sect. 3 , condition $\left(\mathcal{P}_{3}\right)$ implies that the operator $\tau$ is in the limit point case at $x=0$. As a consequence, it admits a unique self-adjoint realization (cfr. (3.2)); in the particular case of the operator associated to (2.8), with the Coulomb potential

$$
V(x)=\frac{\gamma}{x}, \quad \forall x>0, \gamma<0,
$$

condition $\left(\mathcal{P}_{3}\right)$ is satisfied for a larger range of values of $\gamma$ when $\mu_{a} \neq 0$. This means that the presence of an anomalous magnetic moment has a regularizing effect on the radial Dirac operator (cf. also [23, Sect. 5.3.2].

\subsection{Asymptotic estimates}

In this subsection we describe the behaviour of the solutions of (2.1) when $x \rightarrow+\infty$ or $x \rightarrow 0^{+}$; this will be the consequence of some general results on the asymptotic properties of solutions of linear equations (see e.g. [13]). As a first step, let us consider a system of the form

$$
u^{\prime}=C(\lambda) u+U(x, \lambda) u, \quad x \geq 1, \lambda \in \Lambda
$$

where $C(\lambda)$ and $U(x, \lambda)$ are $2 \times 2$ matrix, for every $\lambda \in \Lambda$ and $x \geq 1$. We have the following result:

Proposition 2.2. [13, Th. 1.5.2, Th. 1.8.1, Th. 1.8.2] Let us suppose that for every $\lambda \in \Lambda$ the matrix $C(\lambda)$ has two real eigenvalues $\sigma_{\lambda}^{-}<0<\sigma_{\lambda}^{+}$and let $u_{\lambda}^{-}, u_{\lambda}^{+}$be the eigenvectors associated to $\sigma_{\lambda}^{-}$and $\sigma_{\lambda}^{+}$, respectively. Moreover, let us assume that

$$
\lim _{x \rightarrow+\infty} U(x, \lambda)=0, \quad \forall \lambda \in \Lambda,
$$


and that there exists $q \geq 1$ such that

$$
\int_{1}^{+\infty}\|U(x, \lambda)\|^{q} d x<+\infty, \quad \forall \lambda \in \Lambda .
$$

Then, for every $\lambda \in \Lambda$ system (2.14) has two linearly independent solutions $u_{1, \lambda}$ and $u_{2, \lambda}$ satisfying

$$
\begin{array}{ll}
u_{1, \lambda}(x)=\left(u_{\lambda}^{-}+o(1)\right) e^{\sigma_{\lambda}^{-}(x-1)+\int_{1}^{x} g_{1, \lambda}(t) d t}, & x \rightarrow+\infty \\
u_{2, \lambda}(x)=\left(u_{\lambda}^{+}+o(1)\right) e^{\sigma_{\lambda}^{+}(x-1)+\int_{1}^{x} g_{2, \lambda}(t) d t}, & x \rightarrow+\infty,
\end{array}
$$

where, for $i=1,2$, we have

$$
\begin{array}{ll}
g_{i, \lambda}=0 & \text { if } q=1 \\
g_{i, \lambda} \in L^{q}(1,+\infty) & \text { if } q>1 .
\end{array}
$$

Proof. Let us note that when $q=1$ the result follows from [13, Th. 1.8.1]. Therefore, assume that $q>1$; from [13, Th. 1.5.2, Th. 1.8.2] we immediately deduce that (2.17) is satisfied with some functions $g_{i, \lambda}, i=1,2, \lambda \in \Lambda$, such that

$$
g_{i, \lambda}= \begin{cases}0 & \text { if } q=1 \\ \sum_{m=1}^{M} g_{i, m, \lambda} & \text { if } q>1,\end{cases}
$$

with $M$ such that $2^{M-1}<q \leq 2^{M}$ and

$$
g_{i, m, \lambda} \in L^{q / 2^{m-1}}(1,+\infty), \quad \forall m=1, \ldots, M .
$$

Now, assumption (2.15) implies that

$$
\lim _{x \rightarrow+\infty} g_{i, m, \lambda}(x)=0, \quad \forall m=1, \ldots, M, \lambda \in \Lambda, i=1,2
$$

(see also formula (1.5.27) in [13]). Hence, for every $i=1,2, \lambda \in \Lambda$ and $m=$ $1, \ldots, M$ we have

$$
g_{i, m, \lambda} \in L^{q / 2^{m-1}}(1,+\infty) \Rightarrow g_{i, m, \lambda} \in L^{q}(1,+\infty) .
$$

This implies that $q_{i, \lambda} \in L^{q}(1,+\infty)$, for every $i=1,2$ and $\lambda \in \Lambda$.

Now, let us observe that

$$
f \in L^{p}(1,+\infty), p>1 \Rightarrow\left|\int_{1}^{x} f(t) d t\right| \leq\|f\|_{L^{p}}(x-1)^{1 / p^{\prime}}, \quad \forall x \geq 1,
$$

where $p^{\prime}$ is the conjugate exponent of $p$; noting that in this case $1 / p^{\prime}<1$, from Proposition 2.2 we obtain the following result:

Proposition 2.3. Under the assumptions of Proposition 2.2, for every $\lambda \in \Lambda$ we have

$$
\lim _{x \rightarrow+\infty} u_{1, \lambda}(x)=0
$$

and

$$
\lim _{x \rightarrow+\infty}\left\|u_{2, \lambda}(x)\right\|=+\infty .
$$


Moreover, if $q>1$ in (2.16), then there exists $x_{1}>1$ such that

$$
\sigma_{\lambda}^{-}(x-1)+\int_{1}^{x} g_{1, \lambda}(t) d t \leq \frac{\sigma_{\lambda}^{-}}{2}(x-1), \quad \forall x \geq x_{1} .
$$

Using Proposition 2.2 and Proposition 2.3 we are able to prove some asymptotic results on the solutions of (2.1) when $x \rightarrow+\infty$ or $x \rightarrow 0^{+}$. We start with the study of (2.1) when $x \rightarrow+\infty$ (cf. also [4]); assume then $x \geq 1$.

Let us first observe that $(2.1)$ can be written as

$$
z^{\prime}=B_{\lambda} z+Q(x) z
$$

where

$$
B_{\lambda}=J^{-1}\left(\lambda \operatorname{Id}-P_{\infty}\right), \quad Q(x)=J^{-1}\left(P_{\infty}-P(x)\right), \quad \forall x>0 .
$$

This form corresponds to (2.14) with $C(\lambda)=B_{\lambda}$ and $U(x, \lambda)=Q(x)$, for every $x \geq 1, \lambda \in \Lambda$; note that assumptions (2.2) and (2.3) imply that (2.15) and (2.16), with $q=q_{\infty}$, hold true. Moreover, if $\lambda \in \Lambda$, setting $\Delta_{\lambda}=\left(\mu^{+}-\right.$ $\lambda)\left(\lambda-\mu^{-}\right)$, then $B_{\lambda}$ has the real eigenvalues $\pm \sqrt{\Delta_{\lambda}}$; in this situation we denote by $b_{1, \lambda}=\left(\lambda-\mu^{+}, \sqrt{\Delta_{\lambda}}\right)$ and $b_{2, \lambda}=\left(\mu^{+}-\lambda, \sqrt{\Delta_{\lambda}}\right)$ the eigenvectors of $B_{\lambda}$ associated to the eigenvalues $-\sqrt{\Delta_{\lambda}}$ and $\sqrt{\Delta_{\lambda}}$, respectively.

Therefore from Proposition 2.2 and Proposition 2.3 we deduce the following results:

Proposition 2.4. For every $\lambda \in \Lambda$ system (2.1) has two linearly independent solutions $z_{1, \lambda}$ and $z_{2, \lambda}$ satisfying

$$
\begin{array}{ll}
z_{1, \lambda}(x)=\left(b_{1, \lambda}+o(1)\right) e^{-\sqrt{\Delta_{\lambda}}(x-1)+\int_{1}^{x} g_{1}(t) d t}, & x \rightarrow+\infty \\
z_{2, \lambda}(x)=\left(b_{2, \lambda}+o(1)\right) e^{\sqrt{\Delta_{\lambda}}(x-1)+\int_{1}^{x} g_{2}(t) d t}, & x \rightarrow+\infty,
\end{array}
$$

where, for $i=1,2$, we have

$$
\begin{array}{ll}
g_{i}=0 & \text { if } q_{\infty}=1 \\
g_{i} \in L^{q_{\infty}}(1,+\infty) & \text { if } q_{\infty}>1 .
\end{array}
$$

Lemma 2.5. Assume that $\lambda \in \Lambda$ and let $z_{1, \lambda}$ and $z_{2, \lambda}$ be the solutions of (2.1) given in Proposition 2.4. Then

$$
\lim _{x \rightarrow+\infty} z_{1, \lambda}(x)=0
$$

and

$$
\lim _{x \rightarrow+\infty}\left|\left(z_{2, \lambda}\right)_{1}(x)\right|=\lim _{x \rightarrow+\infty}\left|\left(z_{2, \lambda}\right)_{2}(x)\right|=+\infty .
$$

Moreover, $z_{1, \lambda} \in H^{1}(1,+\infty)$.

Proof. The relations (2.28) and (2.29) immediately follow from (2.22) and (2.23). In particular (2.29) comes from the fact that neither component of $b_{2, \lambda}$ vanishes.

Moreover, from (2.24) we deduce that there exists $K_{1, \lambda}>0$ such that

$$
\left\|z_{1, \lambda}(x)\right\| \leq K_{1, \lambda} e^{-\sqrt{\Delta_{\lambda}}(x-1)}, \quad \forall x \geq x_{1} ;
$$


this implies that $z_{1, \lambda} \in L^{2}(1,+\infty)$. Now, from the differential equation we deduce that

$$
J z_{1, \lambda}^{\prime}(x)=\lambda z_{1, \lambda}(x)-P(x) z_{1, \lambda}(x), \quad \forall x \geq 1 ;
$$

since $P \in L^{\infty}(1,+\infty)$, we infer that $J z_{1, \lambda}^{\prime} \in L^{2}(1,+\infty)$ and then $z_{1, \lambda} \in$ $H^{1}(1,+\infty)$.

Arguing as in the proof of [4, Lemma 2.3], we obtain the following result:

Lemma 2.6. Assume that $\lambda \in \Lambda$ and let $z=(u, v)$ be a nontrivial solution of (2.1). Then either

$$
\lim _{x \rightarrow+\infty} u(x)=\lim _{x \rightarrow+\infty} v(x)=0
$$

or

$$
\lim _{x \rightarrow+\infty}|u(x)|=\lim _{x \rightarrow+\infty}|v(x)|=+\infty .
$$

Moreover, $z \in H^{1}(1,+\infty)$ if and only if (2.30) holds true and there exists $\gamma>0$ such that $z=\gamma z_{1, \lambda}$, where $z_{1, \lambda}$ is given in Proposition 2.4.

Now, let us study the behaviour of the solutions of (2.1) when $x \rightarrow 0^{+}$; assume then that $x \in(0,1)$. For every $\beta \geq 1$ let us consider an invertible function $\phi_{\beta} \in C^{1}((1,+\infty),(0,1))$ such that

$$
\lim _{t \rightarrow+\infty} \phi_{\beta}(t)=0 \quad \text { and } \quad \lim _{t \rightarrow 1^{+}} \phi_{\beta}(t)=1 .
$$

The change of variable $x=\phi_{\beta}(t)$ transforms (2.1) into

$$
w^{\prime}=-J^{-1} P\left(\phi_{\beta}(t)\right) \phi_{\beta}^{\prime}(t) w+\lambda J^{-1} \phi_{\beta}^{\prime}(t) w,
$$

where $w(t)=z\left(\phi_{\beta}(t)\right)$, for every $t \geq 1$. With a suitable choice of $\phi_{\beta}$ system (2.33) can be reduced to a system of the form (2.14):

Lemma 2.7. Assume $\beta=1$ in $\left(\mathcal{P}_{2}\right)$ and let

$$
\phi_{\beta}(t)=e^{1-t}, \quad \forall t \geq 1 \text {. }
$$

Then (2.33) reduces to a system of the form (2.14) with

$$
C !=C(\lambda)=J^{-1} P^{*}, \quad U(t, \lambda)=J^{-1}\left(R_{0}\left(e^{1-t}\right)-\lambda e^{1-t} \mathrm{Id}\right),
$$

for every $t \geq 1$ and $\lambda \in \Lambda$.

Lemma 2.8. Assume $\beta>1$ in $\left(\mathcal{P}_{2}\right)$ and let

$$
\phi_{\beta}(t)=t^{-1 /(\beta-1)}, \quad \forall t \geq 1 .
$$

Then (2.33) reduces to a system of the form (2.14) with

$C=C(\lambda)=\frac{1}{\beta-1} J^{-1} P^{*}, \quad U(t, \lambda)=\frac{1}{\beta-1} J^{-1}\left(R_{0}\left(t^{-1 /(\beta-1)}\right)-\lambda t^{-\beta /(\beta-1)} \mathrm{Id}\right)$,

for every $t \geq 1$ and $\lambda \in \Lambda$. 
The proofs of Lemma 2.7 and Lemma 2.8 are straightforward and therefore they are omitted.

Now, set $\Delta^{*}=-\operatorname{det} P^{*}$ and observe that the matrix $C$ given in (2.34) or (2.35) has the eigenvalues $\sigma^{ \pm}= \pm \sqrt{\Delta^{*}}$ if $\beta=1$ and $\sigma^{ \pm}= \pm \sqrt{\Delta^{*}} /(\beta-1)$ if $\beta>1$; in what follows, we will denote by $w_{1}^{*}$ and $w_{2}^{*}$ the eigenvectors of $C$ associated to $\sigma^{ \pm}$.

Moreover, from (2.4) and the definition of $R_{0}$ we deduce that the function $U$ given in (2.34) or (2.35) satisfies (2.15). Finally, let us note that (2.5) implies that (2.16) is satisfied with $q=q_{0}$; indeed, when $\beta=1$ we have

$$
\begin{gathered}
\int_{1}^{+\infty}\|U(t, \lambda)\|^{q_{0}} d t \leq\left\{\left[\int_{1}^{+\infty}\left\|R_{0}\left(e^{1-t}\right)\right\|^{q_{0}} d t\right]^{\frac{1}{q_{0}}}+\lambda\left[\int_{1}^{+\infty} e^{q_{0}(1-t)} d t\right]^{\frac{1}{q_{0}}}\right\}^{q_{0}} \\
=\left\{\left[\int_{0}^{1} \frac{1}{x}\left\|R_{0}(x)\right\|^{q_{0}} d x\right]^{\frac{1}{q_{0}}}+\frac{\lambda}{q_{0}^{1 / q_{0}}}\right\}^{q_{0}}<+\infty .
\end{gathered}
$$

On the other hand, if $\beta>1$ we deduce that

$$
\begin{aligned}
& \int_{1}^{+\infty}\|U(t, \lambda)\|^{q_{0}} d t \leq \frac{1}{(\beta-1)^{q_{0}}}\left\{\left[\int_{1}^{+\infty}\left\|R_{0}\left(t^{-1 /(\beta-1)}\right)\right\|^{q_{0}} d t\right]^{\frac{1}{q_{0}}}\right. \\
& \left.\quad+\lambda\left[\int_{1}^{+\infty} t^{-\beta q_{0} /(\beta-1)} d t\right]^{\frac{1}{q_{0}}}\right\}^{q_{0}} \\
& \quad=\frac{1}{\beta-1}\left\{\left[(\beta-1) \int_{0}^{1} \frac{1}{x^{\beta}}\left\|R_{0}(x)\right\|^{q_{0}} d x\right]^{\frac{1}{q_{0}}}+\lambda\left(\frac{\beta-1}{q_{0}}\right)^{\frac{1}{q_{0}}}\right\}^{q_{0}}<+\infty .
\end{aligned}
$$

Therefore, we can apply Proposition 2.2 and Proposition 2.3 to (2.33), with $\phi_{\beta}$ as above, and obtain the following results:

Proposition 2.9. For every $\lambda \in \Lambda$ system (2.33), with $\phi_{\beta}$ as in Lemma 2.7 or Lemma 2.8, has two linearly independent solutions $w_{1, \lambda}$ and $w_{2, \lambda}$ satisfying

$$
\begin{aligned}
& w_{1, \lambda}(t)=\left(w_{1}^{*}+o(1)\right) e^{-\sqrt{\Delta^{*}}(t-1)+\int_{1}^{t} g_{1, \lambda}(s) d s}, \quad t \rightarrow+\infty \\
& w_{2, \lambda}(t)=\left(w_{2}^{*}+o(1)\right) e^{\sqrt{\Delta^{*}}(t-1)+\int_{1}^{t} g_{2, \lambda}(s) d s}, \quad t \rightarrow+\infty,
\end{aligned}
$$

if $\beta=1$ and

$$
\begin{aligned}
& w_{1, \lambda}(t)=\left(w_{1}^{*}+o(1)\right) e^{-\frac{\sqrt{\Delta^{*}}}{\beta-1}(t-1)+\int_{1}^{t} g_{1, \lambda}(s) d s,} \quad t \rightarrow+\infty \\
& w_{2, \lambda}(t)=\left(w_{2}^{*}+o(1)\right) e^{\frac{\sqrt{\Delta^{*}}}{\beta-1}(t-1)+\int_{1}^{t} g_{2, \lambda}(s) d s}, \quad t \rightarrow+\infty,
\end{aligned}
$$

if $\beta>1$, where, for $i=1,2$, we have

$$
\begin{array}{ll}
g_{i, \lambda}=0 & \text { if } q_{0}=1 \\
g_{i, \lambda} \in L^{q_{0}}(1,+\infty) & \text { if } q_{0}>1 .
\end{array}
$$


Lemma 2.10. Assume that $\lambda \in \Lambda$ and let $w_{1, \lambda}$ and $w_{2, \lambda}$ be the solutions of (2.33) given in Proposition 2.9. Then

$$
\lim _{t \rightarrow+\infty} w_{1, \lambda}(t)=0
$$

and

$$
\lim _{t \rightarrow+\infty}\left\|w_{2, \lambda}(t)\right\|=+\infty .
$$

Moreover, the solution $w_{1, \lambda}$ satisfies

$$
\int_{1}^{+\infty}\left\|w_{1, \lambda}(t)\right\|^{2} e^{t} d t<+\infty, \quad \text { if } \beta=1
$$

and

$$
\int_{1}^{+\infty}\left\|w_{1, \lambda}(t)\right\|^{2} t^{\beta /(\beta-1)} d t<+\infty, \quad \text { if } \beta>1 .
$$

Proof. Let us note that (2.39) and (2.40) immediately follow from (2.22) and $(2.23)$.

As far as (2.41) is concerned, from (2.36) we deduce that there exists $K_{1, \lambda}>0$ such that

$$
\left\|w_{1, \lambda}(t)\right\|^{2} e^{t} \sim K_{1, \lambda} e^{-2 \sqrt{\Delta^{*}}(t-1)+2 \int_{1}^{t} g_{1, \lambda}(s) d s} e^{t}, \quad t \rightarrow+\infty ;
$$

now, let us observe that $1-2 \sqrt{\Delta^{*}}>0$, since $(2.6)$ holds. Hence, using again (2.21) we infer that there exists $t_{1}>1$ such that

$$
e^{\left(1-2 \sqrt{\Delta^{*}}\right) t+2 \int_{1}^{t} g_{1, \lambda}(s) d s} \leq e^{\left(1-2 \sqrt{\Delta^{*}}\right) t / 2}, \quad \forall t \geq t_{1},
$$

is satisfied. Conditions (2.43) and (2.44) imply (2.41).

Finally, when $\beta>1$ from (2.37) we deduce that there exists $M_{1, \lambda}>0$ such that

$$
\left\|w_{1, \lambda}(t)\right\|^{2} t^{\beta /(\beta-1)} \sim M_{1, \lambda} e^{-2 \frac{\sqrt{\Delta^{*}}}{\beta-1}(t-1)+2 \int_{1}^{t} g_{1, \lambda}(s) d s} t^{\beta /(\beta-1)}, \quad t \rightarrow+\infty
$$

moreover, from (2.24) we infer that there exists $t_{2}>1$ such that

$$
e^{-2 \frac{\sqrt{\Delta^{*}}}{\beta-1}(t-1)+2 \int_{1}^{t} g_{1, \lambda}(s) d s} \leq e^{-\frac{\sqrt{\Delta^{*}}}{\beta-1}(t-1)}, \quad \forall t \geq t_{2},
$$

is satisfied. Conditions (2.45) and (2.46) imply (2.42).

The next result is a consequence of Proposition 2.9 and Lemma 2.10.

Proposition 2.11. For every $\lambda \in \Lambda$ system (2.1) has two linearly independent solutions $\zeta_{1, \lambda}$ and $\zeta_{2, \lambda}$ satisfying

$$
\begin{aligned}
& \zeta_{1, \lambda}(x)=\left(w_{1}^{*}+o(1)\right) x^{\sqrt{\Delta^{*}}} e^{\int_{1}^{1-\log x} g_{1, \lambda}(s) d s}, \quad x \rightarrow 0^{+} \\
& \zeta_{2, \lambda}(x)=\left(w_{2}^{*}+o(1)\right) x^{-\sqrt{\Delta^{*}}} e^{\int_{1}^{1-\log x} g_{2, \lambda}(s) d s}, \quad x \rightarrow 0^{+},
\end{aligned}
$$

if $\beta=1$ and

$$
\begin{aligned}
& \zeta_{1, \lambda}(x)=\left(w_{1}^{*}+o(1)\right) e^{-\frac{\sqrt{\Delta^{*}}}{\beta-1} x^{1-\beta}+\int_{1}^{-\log x} g_{1, \lambda}(s) d s}, \quad x \rightarrow 0^{+} \\
& \zeta_{2, \lambda}(x)=\left(w_{2}^{*}+o(1)\right) e^{\frac{\sqrt{\Delta^{*}}}{\beta-1} x^{1-\beta} \int_{1}^{-\log x} g_{2, \lambda}(s) d s}, \quad x \rightarrow 0^{+},
\end{aligned}
$$


if $\beta>1$, where, for $i=1,2$, we have

$$
\begin{array}{ll}
g_{i, \lambda}=0 & \text { if } q_{0}=1 \\
g_{i, \lambda} \in L^{q_{0}}(1,+\infty) & \text { if } q_{0}>1 .
\end{array}
$$

Lemma 2.12. Assume that $\lambda \in \Lambda$ and let $\zeta_{1, \lambda}$ and $\zeta_{2, \lambda}$ be the solutions of (2.1) given in Proposition 2.11. Then

$$
\lim _{x \rightarrow 0^{+}} \zeta_{1, \lambda}(x)=0
$$

and

$$
\lim _{x \rightarrow 0^{+}}\left\|\zeta_{2, \lambda}(x)\right\|=+\infty .
$$

Moreover, $\zeta_{1, \lambda} \in H^{1}(0,1)$.

Proof. The relations (2.50) and (2.51) immediately follow from (2.39) and $(2.40)$.

Now, assume that $\beta=1$; let us observe that we have

$$
\int_{0}^{1} \frac{\left\|\zeta_{1, \lambda}(x)\right\|^{2}}{x^{2}} d x=\int_{1}^{+\infty}\left\|\zeta_{1, \lambda}\left(e^{1-t}\right)\right\|^{2} e^{t-1} d t=\int_{1}^{+\infty}\left\|w_{1, \lambda}(t)\right\|^{2} e^{t-1} d t<+\infty
$$

by (2.41). This condition obviously implies that

$$
\int_{0}^{1}\left\|\zeta_{1, \lambda}(x)\right\|^{2} d x \leq \int_{0}^{1} \frac{\left\|\zeta_{1, \lambda}(x)\right\|^{2}}{x^{2}} d x<+\infty
$$

and so $\zeta_{1, \lambda} \in L^{2}(0,1)$; in order to prove that $\zeta_{1, \lambda}^{\prime} \in L^{2}(0,1)$, let us note that $\zeta_{1, \lambda}$ satisfies

$$
J \zeta_{1, \lambda}^{\prime}(x)=\lambda \zeta_{1, \lambda}(x)-P(x) \zeta_{1, \lambda}(x), \quad \forall x \in(0,1) .
$$

From $\left(\mathcal{P}_{2}\right)$ we deduce that there exists $K>0$ such that

$$
\|P(x)\| \leq \frac{K}{x}, \quad \forall x \in(0,1) ;
$$

therefore, (2.52) implies that $P \zeta_{1, \lambda} \in L^{2}(0,1)$ and then also $\zeta_{1, \lambda}^{\prime} \in L^{2}(0,1)$.

When $\beta>1$ by (2.42) we obtain

$$
\begin{aligned}
\int_{0}^{1} \frac{\left\|\zeta_{1, \lambda}(x)\right\|^{2}}{x^{2 \beta}} d x & =\frac{1}{\beta-1} \int_{1}^{+\infty} \frac{\left\|\zeta_{1, \lambda}\left(t^{-1 /(\beta-1)}\right)\right\|^{2}}{t^{-2 \beta /(\beta-1)}} t^{-\beta /(\beta-1)} d t \\
& =\int_{1}^{+\infty}\left\|w_{1, \lambda}(t)\right\|^{2} t^{\beta /(\beta-1)} d t<+\infty
\end{aligned}
$$

This condition implies that

$$
\int_{0}^{1}\left\|\zeta_{1, \lambda}(x)\right\|^{2} d x \leq \int_{0}^{1} \frac{\left\|\zeta_{1, \lambda}(x)\right\|^{2}}{x^{2 \beta}} d x<+\infty
$$

and so $\zeta_{1, \lambda} \in L^{2}(0,1)$; arguing as above, in order to prove that $\zeta_{1, \lambda}^{\prime} \in L^{2}(0,1)$, let us note that $\zeta_{1, \lambda}$ satisfies

$$
J \zeta_{1, \lambda}^{\prime}(x)=\lambda \zeta_{1, \lambda}(x)-P(x) \zeta_{1, \lambda}(x), \quad \forall x \in(0,1) .
$$


From $\left(\mathcal{P}_{2}\right)$ we deduce that there exists $M>0$ such that

$$
\|P(x)\| \leq \frac{M}{x^{\beta}}, \quad \forall x \in(0,1) ;
$$

therefore, (2.54) implies that $P \zeta_{1, \lambda} \in L^{2}(0,1)$ and then also $\zeta_{1, \lambda}^{\prime} \in L^{2}(0,1)$.

Lemma 2.13. Assume that $\lambda \in \Lambda$ and let $z=(u, v)$ be a nontrivial solution of (2.1). Then either

$$
\lim _{x \rightarrow 0^{+}} u(x)=\lim _{x \rightarrow 0^{+}} v(x)=0
$$

or

$$
\lim _{x \rightarrow 0^{+}}\|z(x)\|=+\infty .
$$

Moreover, $z \in H^{1}(0,1)$ if and only if (2.56) holds true and there exists $\xi \in \mathbb{R}$ such that $z=\xi \zeta_{1, \lambda}$, where $\zeta_{1, \lambda}$ is given in Proposition 2.11.

Remark 2.14. Let us denote by $Z$ the set of solutions of (2.1). From Proposition 2.4 and Proposition 2.11 we deduce that

$$
Z=\operatorname{span}\left\{z_{1, \lambda}, z_{2, \lambda}\right\}=\operatorname{span}\left\{\zeta_{1, \lambda}, \zeta_{2, \lambda}\right\}
$$

As far as nontrivial solutions $z \in L^{2}(0,+\infty)$ are concerned, let us observe that Lemma 2.6 and Lemma 2.13 prove that

$$
z \in L^{2}(1,+\infty) \quad \Longleftrightarrow \quad z \in \operatorname{span}\left\{z_{1, \lambda}\right\}:=Z_{\infty}
$$

and

$$
z \in L^{2}(0,1) \quad \Longleftrightarrow \quad z \in \operatorname{span}\left\{\zeta_{1, \lambda}\right\}:=Z_{0} .
$$

As a consequence, $z \in L^{2}(0,+\infty)$ is a solution of (2.1) if and only if

$$
z \in Z_{0} \cap Z_{\infty} .
$$

We conclude this subsection with some explicit formulas for solutions of the non-homogeneous equation

$$
J z^{\prime}+P(x) z=f,
$$

where $z, f \in L^{2}(0,1)$. They are based on the fact that the homogeneous equation (2.1) has a suitable dichotomy at zero when $\lambda=0$ (see [6]).

First of all, let us observe that from $[6, \S 3]$ we deduce that $(2.58)$ has a solution $z_{f} \in L^{\infty}(0,1)$ when $f \in L^{2}(0,1)$. Moreover, let us point out that the previous results on the asymptotic behaviour for $x \rightarrow 0^{+}$of the solutions of (2.1) hold true also when $\lambda=0$; indeed, they are based on the fact that $\Delta^{*}>0$. Hence, according to Remark 2.14, all the solutions $z \in L^{2}(0,1)$ of (2.58) are of the form

$$
z=c \zeta_{1,0}+z_{f}
$$

for some $c \in \mathbb{R}$. More precisely, we have the following result: 
Theorem 2.15. [6, §3] Let us consider $f \in L^{2}(0,1)$ and let $z \in L^{2}(0,1)$ be a solution of (2.58). Then, there exist $c \in \mathbb{R}$ and $G:(0,1) \times(0,1) \longrightarrow \mathbb{R}^{2}$ such that

$$
z(x)=c \zeta_{1,0}(x)+\int_{0}^{1} G(x, \xi) f(\xi) d \xi, \quad \forall x \in(0,1) .
$$

Moreover, there exist $K>0$ such that

$$
\begin{aligned}
& \|G(x, \xi)\| \\
& \leq\left\{\begin{array}{ll}
K\left(\frac{\min (x, \xi)}{\max (x, \xi)}\right)^{\sqrt{\Delta^{*}}} & \text { if } \beta=1 \\
K\left(\frac{e^{-\min (x, \xi)^{1-\beta}}}{e^{-\max (x, \xi)^{1-\beta}}}\right)^{\sqrt{\Delta^{*}} /(\beta-1)} & \text { if } \beta>1
\end{array} \leq K, \quad \forall(x, \xi) \in(0,1) \times(0,1) .\right.
\end{aligned}
$$

Proof. We just point out that the result follows from the change of variables $x=\phi_{\beta}(t)$, from estimates in $[6, \S 3$, formulas (3) and (4)] and Propositions 2.9 or 2.11 .

\subsection{Oscillatory properties}

In this subsection we develop an oscillatory theory for nontrivial solutions of (2.1), based on the study of the angular coordinate in the phase-plane (see $[24])$. For every nontrivial solution $(u, v, \lambda)$ of $(2.1)$ let us introduce the polar coordinates $(\rho, \theta)=(\rho(x, \lambda), \theta(x, \lambda))$ according to

$$
\left\{\begin{array}{l}
u=\rho \cos \theta \\
v=\rho \sin \theta
\end{array}\right.
$$

Observe that $\theta$ is defined mod. $2 \pi$; we do not impose a normalization condition on $\theta$ and then the following results hold true for any angular coordinate associated to a nontrivial solution $z$. As a first step, we are able to study the asymptotic behaviour of $\theta$ when $x \rightarrow+\infty$ or $x \rightarrow 0^{+}$; this follows from the results of Subsection 2.1 .

Proposition 2.16. [4, Prop. 2.4] For every $\lambda \in \Lambda$ the function $\theta(\cdot, \lambda)$ has limit at infinity and we have either

$$
\lim _{x \rightarrow+\infty} \theta(x, \lambda)=\pi-\arctan \sqrt{\frac{\lambda-\mu^{-}}{\mu^{+}-\lambda}}(\bmod \pi)
$$

or

$$
\lim _{x \rightarrow+\infty} \theta(x, \lambda)=\arctan \sqrt{\frac{\lambda-\mu^{-}}{\mu^{+}-\lambda}}(\bmod \pi) .
$$

Moreover, (2.61) and (2.62) correspond to the cases when (2.30) and (2.31) are fulfilled, respectively. 
Proposition 2.17. For every $\lambda \in \Lambda$ the function $\theta(\cdot, \lambda)$ has limit at zero and we have either

$$
\lim _{x \rightarrow 0^{+}} \theta(x, \lambda)=\arctan \frac{w_{1,2}^{*}}{w_{1,1}^{*}} \quad(\bmod \pi)
$$

or

$$
\lim _{x \rightarrow 0^{+}} \theta(x, \lambda)=\arctan \frac{w_{2,2}^{*}}{w_{2,1}^{*}} \quad(\bmod \pi),
$$

where $w_{1}^{*}$ and $w_{2}^{*}$ are eigenvectors of $C$ associated to the eigenvalues $\sigma^{ \pm}$, respectively. Moreover, (2.63) and (2.64) correspond to the cases when (2.56) and (2.57) are fulfilled, respectively.

Let us observe that the possible limits of $\theta(\cdot, \lambda)$ at zero do not depend on $\lambda \in \Lambda$; in what follows, we denote

$$
\begin{aligned}
& \theta(+\infty, \lambda)=\lim _{x \rightarrow+\infty} \theta(x, \lambda) \\
& \theta(0)=\lim _{x \rightarrow 0^{+}} \theta(x, \lambda),
\end{aligned}
$$

which exist and are finite by Proposition 2.16 and Proposition 2.17.

Remark 2.18. According to Remark 2.14 and the above Propositions, we deduce that for a nontrivial solution $z$ of (2.1) we have

$$
z \in L^{2}(1,+\infty) \Longleftrightarrow \theta(+\infty, \lambda)=\pi-\arctan \sqrt{\frac{\lambda-\mu^{-}}{\mu^{+}-\lambda}}(\bmod \pi)
$$

and

$$
z \in L^{2}(0,1) \Longleftrightarrow \theta(0)=\arctan \frac{w_{1,2}^{*}}{w_{1,1}^{*}} \quad(\bmod \pi) .
$$

Proposition 2.16 and Proposition 2.17 imply that any angular function $\theta(\cdot, \lambda)$ is bounded on $(0,+\infty)$, for every $\lambda \in \Lambda$. As a consequence, we can associate to every nontrivial solution $z$ of $(2.1)$ the rotation number

$$
\operatorname{rot}(z)=\frac{\theta(+\infty, \lambda)-\theta(0)}{\pi}
$$

Roughly speaking, the unboundedness of the interval and the singularity at zero do not prevent solutions to perform only a finite number of rotations around the origin (as in the regular case). It is important to observe that rot $(z)$ does not depend on the choice of the angular function of $z$. In Sect. 4 we will study some continuity properties of the rotation number defined in (2.65).

We conclude this subsection with some asymptotic phase-plane analysis for (2.1); as above, we prove the results for $x \rightarrow+\infty$. The case of $x \rightarrow 0^{+}$can be obtained in an analogous way by means of the change of variable $x=\phi_{\beta}(t)$ already introduced.

Let us consider again (2.25), which is equivalent to (2.1), and a similar system

$$
J z^{\prime}+\tilde{P}(x) z=\tilde{\lambda} z
$$


where $\tilde{P} \in \mathcal{P}_{\mu}$ and $\tilde{\lambda} \in \Lambda ;(2.66)$ can be written in the form

$$
z^{\prime}=B_{\tilde{\lambda}} z+\tilde{Q}(x) z
$$

where $B_{\tilde{\lambda}}=J^{-1}\left(\tilde{\lambda} \mathrm{Id}-P_{\infty}\right)$ and $\tilde{Q}(x)=J^{-1}\left(P_{\infty}-\tilde{P}(x)\right)$, for every $x>0$. Let us note that the matrix $P_{\infty}$ is the same both for $P$ and $\tilde{P}$, since $P, \tilde{P} \in \mathcal{P}_{\mu}$.

For every $\lambda \in \Lambda$, let $b_{1, \lambda}, b_{2, \lambda}$ be as in Proposition 2.4; from the discussion leading to Proposition 2.4 we know that

$$
b_{1, \lambda}=\left(\lambda-\mu^{+}, \sqrt{\Delta_{\lambda}}\right), \quad b_{2, \lambda}=\left(-\lambda+\mu^{+}, \sqrt{\Delta_{\lambda}}\right),
$$

for every $\lambda \in \Lambda$; moreover, there exists $\rho_{\lambda}>0$ such that

$$
\begin{aligned}
& b_{1, \lambda}=\rho_{\lambda}\left(\cos \theta_{\infty, \lambda}, \sin \theta_{\infty, \lambda}\right) \\
& b_{2, \lambda}=\rho_{\lambda}\left(-\cos \theta_{\infty, \lambda}, \sin \theta_{\infty, \lambda}\right),
\end{aligned}
$$

where

$$
\theta_{\infty, \lambda}=\pi-\arctan \sqrt{\frac{\lambda-\mu^{-}}{\mu^{+}-\lambda}}(\bmod \pi) .
$$

For every $\theta \in \mathbb{R}$, let $r_{\theta}$ be the straight line of equation $x \sin \theta-y \cos \theta=0$ and let $v_{\theta}=(\sin \theta,-\cos \theta)$; moreover, let $r_{\theta}^{ \pm}$be the half-lines given by the intersection of $r_{\theta}$ with the half-planes $H^{+}=\left\{(x, y) \in \mathbb{R}^{2}: \quad x>0\right\}$ and $H^{-}=\left\{(x, y) \in \mathbb{R}^{2}: x<0\right\}$, respectively. We are in position to prove the following result:

Proposition 2.19. For every $\tilde{\lambda} \in \Lambda, \tilde{P} \in \mathcal{P}_{\mu}$ and for every $\theta \in(\pi / 2, \pi)$ there exist $\tilde{\delta}>0$ and $\tilde{x}_{\infty}=\tilde{x}_{\infty}(\tilde{\lambda}, \tilde{P}, \theta)>0$ such that for every $\lambda \in \Lambda$ and $P \in \mathcal{P}_{\mu}$ with

$$
|\lambda-\tilde{\lambda}|<\tilde{\delta}, \quad\|P-\tilde{P}\|_{L^{\infty}(1,+\infty)}<\tilde{\delta}
$$

we have

$$
\begin{aligned}
& \theta<\theta_{\infty, \lambda} \quad \Rightarrow \quad\left\langle v_{\theta}, B_{\lambda} w+Q(x) w\right\rangle>0, \quad \forall w \in r_{\theta}^{-}, \forall x \geq \tilde{x}_{\infty} \\
& \theta>\theta_{\infty, \lambda} \quad \Rightarrow \quad\left\langle v_{\theta}, B_{\lambda} w+Q(x) w\right\rangle<0, \quad \forall w \in r_{\theta}^{-}, \forall x \geq \tilde{x}_{\infty} .
\end{aligned}
$$

Proof. First of all, let us observe that it is sufficient to prove (2.69) when $w$ is a versor. Therefore, let $w=(\cos \theta, \sin \theta)$; a simple computation shows that

$$
\phi_{\lambda}(\theta, w):=\left\langle v_{\theta}, B_{\lambda} w\right\rangle=\cos ^{2} \theta\left(\left(\mu^{+}-\lambda\right) \tan ^{2} \theta-\left(\lambda-\mu^{-}\right)\right), \quad \forall \lambda \in \Lambda .
$$

Let us fix $\tilde{\lambda} \in \Lambda, \tilde{P} \in \mathcal{P}_{\mu}$ and $\theta \in(\pi / 2, \pi)$ such that $\theta<\theta_{\infty, \tilde{\lambda}}$; the continuity of $\theta_{\infty, \lambda}$ as a function of $\lambda \in \Lambda$ implies that there exists $\delta_{1}>0$ such that $\theta<\theta_{\infty, \lambda}$ if $|\lambda-\tilde{\lambda}|<\delta_{1}$.

From (2.70) we deduce that

$$
\begin{aligned}
& \phi_{\tilde{\lambda}}\left(\theta_{\infty, \tilde{\lambda}}, w\right)=0 \\
& \theta<\theta_{\infty, \tilde{\lambda}} \Rightarrow \phi_{\tilde{\lambda}}(\theta, w)>\phi_{\tilde{\lambda}}\left(\theta_{\infty, \tilde{\lambda}}, w\right)=0 .
\end{aligned}
$$


Hence, there exists $\delta_{2} \in\left(0, \delta_{1}\right)$ such that

$$
|\lambda-\tilde{\lambda}|<\delta_{2} \Rightarrow \theta<\theta_{\infty, \lambda} \quad \text { and } \quad \phi_{\lambda}(\theta, w)>\frac{\phi_{\tilde{\lambda}}(\theta, w)}{4}>0
$$

Now, from assumption (2.2) we deduce that

$$
\lim _{x \rightarrow+\infty}\left\langle v_{\theta}, \tilde{Q}(x) w\right\rangle=\lim _{x \rightarrow+\infty}\left\langle v_{\theta}, J^{-1}\left(P_{\infty}-\tilde{P}(x)\right) w\right\rangle=0
$$

this implies that there exists $\tilde{x}_{\infty}=\tilde{x}_{\infty}(\tilde{\lambda}, \tilde{P}, \theta)>1$ such that

$$
x \geq \tilde{x}_{\infty} \Rightarrow\left|\left\langle v_{\theta}, \tilde{Q}(x) w\right\rangle\right|<\frac{\phi_{\tilde{\lambda}}(\theta, w)}{16} .
$$

On the other hand, setting $\delta_{3}=\phi_{\tilde{\lambda}}(\theta, w) / 16$, if $\|P-\tilde{P}\|_{L^{\infty}(1,+\infty)}<\delta_{3}$ we have

$$
\begin{aligned}
& \left|\left\langle v_{\theta}, Q(x) w\right\rangle-\left\langle v_{\theta}, \tilde{Q}(x) w\right\rangle\right|=\left|\left\langle v_{\theta}, J^{-1}(\tilde{P}(x)-P(x)) w\right\rangle\right| \\
& \quad \leq\|\tilde{P}(x)-P(x)\|<\frac{\phi_{\tilde{\lambda}}(\theta, w)}{16}, \quad \forall x \geq 1 .
\end{aligned}
$$

From (2.72) and (2.73) we deduce that

$$
\|P-\tilde{P}\|_{L^{\infty}(1,+\infty)}<\delta_{3}, \quad x \geq \tilde{x}_{\infty} \Rightarrow\left|\left\langle v_{\theta}, Q(x) w\right\rangle\right|<\frac{\phi_{\tilde{\lambda}}(\theta, w)}{8} .
$$

Now, let us set $\tilde{\delta}=\min \left(\delta_{2}, \delta_{3}\right)$; when $|\lambda-\tilde{\lambda}|<\tilde{\delta}$ and $\|P-\tilde{P}\|_{L^{\infty}(1,+\infty)}<\tilde{\delta}$ both (2.71) and (2.74) hold true. As a consequence, we obtain

$$
x \geq \tilde{x}_{\infty} \Rightarrow\left\langle v_{\theta}, B_{\lambda} w+Q(x) w\right\rangle>\frac{\phi_{\tilde{\lambda}}(\theta, w)}{4}-\frac{\phi_{\tilde{\lambda}}(\theta, w)}{8}>0,
$$

i.e. the first inequality in (2.69) is satisfied.

An analogous argument proves the validity of the second inequality in (2.69).

In a very similar way it is possible to prove the following Proposition:

Proposition 2.20. For every $\tilde{\lambda} \in \Lambda, \tilde{P} \in \mathcal{P}_{\mu}$ and for every $\theta \in(0, \pi / 2)$ there exist $\tilde{\delta}_{1}>0$ and $\tilde{x}_{\infty, 1}=\tilde{x}_{\infty, 1}(\tilde{\lambda}, \tilde{P}, \theta)>0$ such that for every $\lambda \in \Lambda$ and $P \in \mathcal{P}_{\mu}$ with

$$
|\lambda-\tilde{\lambda}|<\tilde{\delta}, \quad\|P-\tilde{P}\|_{L^{\infty}(1,+\infty)}<\tilde{\delta}
$$

we have

$$
\begin{aligned}
& \theta<\pi-\theta_{\infty, \lambda} \quad \Rightarrow \quad\left\langle v_{\theta}, B_{\lambda} w+Q(x) w\right\rangle<0, \quad \forall w \in r_{\theta}^{+}, \forall x \geq \tilde{x}_{\infty, 1} \\
& \theta>\pi-\theta_{\infty, \lambda} \quad \Rightarrow \quad\left\langle v_{\theta}, B_{\lambda} w+Q(x) w\right\rangle>0, \quad \forall w \in r_{\theta}^{+}, \forall x \geq \tilde{x}_{\infty, 1} .
\end{aligned}
$$

From Proposition 2.19 and Proposition 2.20 we deduce the following result: 
Proposition 2.21. For every $\tilde{\lambda} \in \Lambda$ and $\tilde{P} \in \mathcal{P}_{\mu}$ there exists $\tilde{\epsilon}>0$ such that for every $\epsilon \in(0, \tilde{\epsilon})$ there exist $\tilde{\delta}>0$ and $\tilde{x}_{\infty}=\tilde{x}_{\infty}(\tilde{\lambda}, \tilde{P}, \epsilon)>0$ such that for every $\lambda \in \Lambda$ and $P \in \mathcal{P}_{\mu}$ with

$$
|\lambda-\tilde{\lambda}|<\tilde{\delta}, \quad\|P-\tilde{P}\|_{L^{\infty}(1,+\infty)}<\tilde{\delta}
$$

and for every nontrivial solution $z \in L^{2}((1,+\infty))$ of $(2.1)$ we have

$$
|\theta(x, \lambda)-\theta(+\infty, \lambda)|<\epsilon, \quad \forall x \geq \tilde{x}_{\infty},
$$

where $\theta(\cdot, \lambda)$ is any angular coordinate of $z$.

Proof. Without loss of generality let us assume that

$$
\theta_{\infty, \lambda}=\pi-\arctan \sqrt{\frac{\lambda-\mu^{-}}{\mu^{+}-\lambda}} \in\left(\frac{\pi}{2}, \pi\right)
$$

and define

$$
\tilde{\epsilon}=\min \left\{\theta_{\infty, \tilde{\lambda}}-\frac{\pi}{2}, \pi-\theta_{\infty, \tilde{\lambda}}\right\}>0 .
$$

Fix any $\epsilon \in(0, \tilde{\epsilon})$ and consider

$$
\theta_{1}=\theta_{\infty, \tilde{\lambda}}-\frac{\epsilon}{2} \quad \text { and } \quad \theta_{2}=\theta_{\infty, \tilde{\lambda}}+\frac{\epsilon}{2},
$$

thus the cone between $r_{\theta_{1}}$ and $r_{\theta_{2}}$ lies inside the II and the IV quadrants and its angular amplitude is exactly $\epsilon$. We use the continuity of $\theta_{\infty, \lambda}$ with respect to $\lambda$ and apply Proposition 2.19 twice with the choices $\theta=\theta_{1}$ and $\theta=\theta_{2}$ in order to find $\tilde{\delta}>0$ and $\tilde{x}_{\infty}=\tilde{x}_{\infty}(\tilde{\lambda}, \tilde{P}, \epsilon)>0$ in such a way that, if (2.78) hold, then $\left|\theta_{\infty, \lambda}-\theta_{\infty, \tilde{\lambda}}\right|<\epsilon / 2$ and (2.69) hold. We remark that $\tilde{x}_{\infty}$ depends only on $\tilde{\lambda}, \tilde{P}, \epsilon$ since the number $\tilde{x}_{\infty, 1}$ provided by Proposition 2.19 depends on $\theta_{1}$ and $\theta_{2}$ which depend only on $\tilde{\lambda}$ and $\epsilon$.

By construction we have $\theta_{1}<\theta_{\infty, \lambda}<\theta_{2}$ and (2.69) implies that the vector field of (2.25) points strictly outwards the cone between $r_{\theta_{1}}$ and $r_{\theta_{2}}$ for all $x \geq \tilde{x}_{\infty}$. Therefore, any nontrivial solution $z \in L^{2}(1,+\infty)$ of $(2.25)$ approaches the origin at the angle $\theta_{\infty, \lambda}$ as $x$ tends to infinity and a standard phase plane argument shows that $z(x)$ must remain inside the cone between $r_{\theta_{1}}$ and $r_{\theta_{2}}$ for all $x \geq \tilde{x}_{\infty}$. Hence (2.79) follows.

By means of the transformation $x=\phi_{\beta}(t)$, it is possible to prove an analogous result concerning the local behaviour of the angular coordinate when $x \rightarrow 0^{+}$; indeed, we have the following:

Proposition 2.22. There exist $\epsilon_{0}>0$ such that for every $\epsilon \in\left(0, \epsilon_{0}\right), \tilde{\lambda} \in \Lambda$ and $\tilde{P} \in \mathcal{P}_{\mu}$ there exist $\tilde{\delta}_{0}>0$ and $x_{0}=x_{0}(\tilde{P}, \epsilon)>0$ such that for every $\lambda \in \Lambda$ and $P \in \mathcal{P}_{\mu}$ with

$$
|\lambda-\tilde{\lambda}|<\tilde{\delta}_{0}, \quad \| P-\left.\tilde{P}\right|_{L^{\infty}(0,1)}<\tilde{\delta}_{0}
$$

and for every nontrivial solution $z \in L^{2}((0,1))$ of $(2.1)$ we have

$$
|\theta(x, \lambda)-\theta(0)|<\epsilon, \quad \forall x \in\left(0, x_{0}\right],
$$

where $\theta(\cdot, \lambda)$ is any angular coordinate of $z$. 


\section{The linear eigenvalue problem}

In this Section we are dealing with the study of the spectral theory for the linear operator formally defined by

$$
\tau z=J z^{\prime}+P(x) z, \quad x>0,
$$

where $P \in \mathcal{P}_{\mu}$. Some information on the spectrum of $\tau$ follow directly from a standard spectral theory (see e.g. $[18,24]$ ). Indeed, $[24$, Th. 6.8] ensures that $\tau$ is in the limit point case at infinity; moreover, from Remark 2.14 we deduce that $\tau$ is in the limit point case also at zero. Let us point out that this fact is a consequence of assumption $\left(\mathcal{P}_{3}\right)$ on $P^{*}$.

Let us consider the operator $A_{0}$ defined by

$$
\begin{aligned}
& D\left(A_{0}\right)=\left\{z \in L^{2}(0,+\infty): z \in A C(0,+\infty), \tau z \in L^{2}(0,+\infty)\right\}, \\
& A_{0} z=\tau z, \quad \forall z \in D\left(A_{0}\right) .
\end{aligned}
$$

From [24, Th. 5.8] we deduce that $A_{0}$ is the unique self-adjoint realization of $\tau$; moreover, arguing as in the proof of [18, Lemma 5.1], it is possible to see that $\sigma_{\text {ess }}\left(A_{0}\right)=\left(-\infty, \mu^{-}\right] \cup\left[\mu^{+},+\infty\right)$. result:

As far as $D_{0}:=D\left(A_{0}\right)$ is concerned, we are able to prove the following

Proposition 3.1. For every $z \in D_{0}$ we have

$$
z \in H^{1}(1,+\infty), \quad z \in L^{\infty}(0,+\infty) .
$$

Proof. Assume that $z \in D_{0}$. Since $P \in L^{\infty}(1,+\infty)$ we deduce that $P(x) z \in$ $L^{2}(1,+\infty)$; hence $J z^{\prime}=\tau z-P(x) z \in L^{2}(1,+\infty)$. This proves that $z \in$ $H^{1}(1,+\infty) \subset L^{\infty}(1,+\infty)$.

The fact that $z \in L^{\infty}(0,1)$ immediately follows from (2.59) and (2.60).

The aim of this Section is to study the problem of the existence of eigenvalues of $A_{0}$ in $\Lambda$; first of all, let us observe that every eigenvalue of $A_{0}$ is simple, since $\tau$ is in the limit point case at infinity. Moreover, from Remark 2.14 we know that $\lambda \in \Lambda$ is an eigenvalue of $A_{0}$ if and only if there exists $c_{\lambda} \in \mathbb{R}$ such that

$$
\zeta_{1, \lambda}=c_{\lambda} z_{1, \lambda}
$$

where $z_{1, \lambda}$ and $\zeta_{1, \lambda}$ are given in Proposition 2.4 and Proposition 2.11, respectively.

Remark 3.2. According to Lemma 2.6 and Lemma 2.13, when $\lambda \in \Lambda$ is an eigenvalue of $A_{0}$ the associated eigenfunction $z_{\lambda}$ satisfies $z_{\lambda} \in H_{0}^{1}(0,+\infty)$.

In what follows we show that it is possible to write a condition equivalent to (3.3) by means of the angular function $\theta$ associated to solutions of (2.1) introduced in Subsection 2.2. To this aim, let us denote by $\vartheta(\cdot, \lambda)$ the angular coordinate of $\zeta_{1, \lambda}$, normalized in such a way that $\vartheta(0) \in(0, \pi)$, for every $\lambda \in \Lambda$.

From Proposition 2.16 we know that there exists

$$
\lim _{x \rightarrow+\infty} \vartheta(x, \lambda)=\vartheta(+\infty, \lambda)
$$


and that this limit corresponds to a function belonging to $H^{1}(1,+\infty)$ if and only if

$$
\vartheta(+\infty, \lambda)=\pi-\arctan \sqrt{\frac{\lambda-\mu^{-}}{\mu^{+}-\lambda}}(\bmod \pi) .
$$

Let us define $\nu: \Lambda \rightarrow \mathbb{R}$ by

$$
\nu(\lambda)=\lim _{x \rightarrow+\infty} \vartheta(x, \lambda), \quad \forall \lambda \in \Lambda .
$$

We then have the following characterization of the eigenvalues of $A_{0}$ :

Theorem 3.3. A number $\lambda \in \Lambda$ is an eigenvalue of $A_{0}$ if and only if

$$
\nu(\lambda)=\pi-\arctan \sqrt{\frac{\lambda-\mu^{-}}{\mu^{+}-\lambda}}(\bmod \pi) .
$$

In order to prove the existence of eigenvalues of $A_{0}$ it is then sufficient to study the behaviour of the function $\nu^{*}: \Lambda \rightarrow \mathbb{R}$ defined by

$$
\nu^{*}(\lambda)=\nu(\lambda)+\arctan \sqrt{\frac{\lambda-\mu^{-}}{\mu^{+}-\lambda}}, \quad \forall \lambda \in \Lambda .
$$

We will prove that $\nu^{*}$ is strictly increasing and continuous in $\Lambda$.

Proposition 3.4. The function $\nu^{*}: \Lambda \rightarrow \mathbb{R}$ is strictly increasing in $\Lambda$.

Proof. Let us first observe that $\nu^{*}$ is the sum of $\nu$ and of the function $\nu_{*}$ defined by

$$
\nu_{*}(\lambda)=\arctan \sqrt{\frac{\lambda-\mu^{-}}{\mu^{+}-\lambda}}, \quad \forall \lambda \in \Lambda ;
$$

since $\nu_{*}$ is strictly increasing in $\Lambda$, it is sufficient to prove that $\nu$ is increasing in $\Lambda$.

To this aim, let us recall (cf. [24, Cor. 16.2]) that for every fixed $x>0$ the function

$$
\begin{aligned}
\varphi_{x}: \Lambda & \rightarrow \mathbb{R} \\
\lambda & \rightarrow \vartheta(x, \lambda)
\end{aligned}
$$

is increasing in $\Lambda$.

Now, let $\lambda, \lambda^{\prime} \in \Lambda$ with $\lambda<\lambda^{\prime}$; for every $x>0$ we have

$$
\vartheta(x, \lambda) \leq \vartheta\left(x, \lambda^{\prime}\right)
$$

passing to the limit for $x \rightarrow+\infty$ we obtain

$$
\lim _{x \rightarrow+\infty} \vartheta(x, \lambda) \leq \lim _{x \rightarrow+\infty} \vartheta\left(x, \lambda^{\prime}\right)
$$

i.e.

$$
\nu(\lambda) \leq \nu\left(\lambda^{\prime}\right)
$$

Proposition 3.5. The function $\nu^{*}: \Lambda \rightarrow \mathbb{R}$ is continuous. 
Proof. Let us observe again that it is sufficient to prove the continuity of $\nu$. To this aim, let us fix $\tilde{\lambda} \in \Lambda$; let us consider $\epsilon>0$ sufficiently small and apply Proposition 2.21 and Proposition 2.22 with $\tilde{P}=P$. Let $\delta_{1}=\min \left(\tilde{\delta}, \tilde{\delta}_{0}\right)$ and let us denote by $x_{\infty}$ and $x_{0}$ the numbers given in those Propositions.

Let us recall that a usual continuous dependence argument on the interval $\left[x_{0}, x_{\infty}\right]$, on which the equation (2.1) is not singular, proves that there exists $\delta_{2}>0$ such that if $|\lambda-\tilde{\lambda}|<\delta_{2}$ then

$$
\left|\left(\vartheta\left(x_{\infty}, \lambda\right)-\vartheta\left(x_{0}, \lambda\right)\right)-\left(\vartheta\left(x_{\infty}, \tilde{\lambda}\right)-\vartheta\left(x_{0}, \tilde{\lambda}\right)\right)\right|<\epsilon
$$

Consider now $\delta=\min \left(\delta_{1}, \delta_{2}\right)$ and assume that $|\lambda-\tilde{\lambda}|<\delta$; we can write

$$
\begin{aligned}
& \nu(\lambda)-\nu(\tilde{\lambda})=\vartheta(+\infty, \lambda)-\vartheta(+\infty, \tilde{\lambda})=\vartheta(+\infty, \lambda)-\vartheta\left(x_{\infty}, \lambda\right) \\
& +\vartheta\left(x_{\infty}, \lambda\right)-\vartheta\left(x_{0}, \lambda\right)+\vartheta\left(x_{0}, \tilde{\lambda}\right)-\vartheta\left(x_{\infty}, \tilde{\lambda}\right)+\vartheta\left(x_{0}, \lambda\right)-\vartheta(0, \lambda) \\
& \quad-\vartheta\left(x_{0}, \tilde{\lambda}\right)+\vartheta(0, \tilde{\lambda})+\vartheta\left(x_{\infty}, \tilde{\lambda}\right)-\vartheta(+\infty, \tilde{\lambda}),
\end{aligned}
$$

taking into account that $\vartheta(0, \lambda)=\vartheta(0, \tilde{\lambda})$. From Proposition 2.21 and Proposition 2.22 we deduce that

$$
\begin{aligned}
& \left|\vartheta(+\infty, \lambda)-\vartheta\left(x_{\infty}, \lambda\right)\right|<\epsilon, \quad\left|\vartheta\left(x_{\infty}, \tilde{\lambda}\right)-\vartheta(+\infty, \tilde{\lambda})\right|<\epsilon \\
& \left|\vartheta\left(x_{0}, \lambda\right)-\vartheta(0, \lambda)\right|<\epsilon, \quad\left|\vartheta\left(x_{0}, \tilde{\lambda}\right)-\vartheta(0, \tilde{\lambda})\right|<\epsilon .
\end{aligned}
$$

From (3.6), (3.7) and (3.8) we obtain

$$
|\nu(\lambda)-\nu(\tilde{\lambda})|<5 \epsilon
$$

and this concludes the proof.

For every $k \in \mathbb{Z}$, let us denote by $\lambda_{k} \in \Lambda$ (if it exists) the number such that

$$
\nu^{*}\left(\lambda_{k}\right)=k \pi
$$

i.e.

$$
\vartheta\left(+\infty, \lambda_{k}\right)=k \pi+\pi-\arctan \sqrt{\frac{\lambda_{k}-\mu^{-}}{\mu^{+}-\lambda_{k}}} .
$$

The number $\lambda_{k}$ is the ' $k$-th eigenvalue' of $A_{0}$ (if it exists) and we denote by $z_{k} \in D_{0}$ the corresponding eigenfunction; recalling (2.63), (2.65), from (3.9) and the fact that $\nu^{*}$ is strictly increasing we immediately deduce the following result:

Proposition 3.6. For every $k \in \mathbb{Z}$ we have

$$
\begin{aligned}
& \operatorname{rot}\left(z_{k}\right) \in(k, k+1) \quad \text { if } \arctan \frac{w_{1,2}^{*}}{w_{1,1}^{*}} \in(0, \pi / 2) \\
& \operatorname{rot}\left(z_{k}\right) \in(k-1 / 2, k+1 / 2) \quad \text { if } \arctan \frac{w_{1,2}^{*}}{w_{1,1}^{*}} \in(\pi / 2, \pi) .
\end{aligned}
$$


Moreover, for every $k, l \in \mathbb{Z}$ with $k \neq l$ we also have

$$
\operatorname{rot}\left(z_{k}\right) \neq \operatorname{rot}\left(z_{l}\right) \text {. }
$$

In what follows, we give some results on the accumulation of eigenvalues of $A_{0}$ at the boundary of $\Lambda$. We consider the (possible) accumulation at the endpoint $\mu^{+}$; conditions for accumulation at $\mu^{-}$can be obtained in an analogous way.

From (3.5) and the definition of $\nu^{*}$ we infer that the existence of eigenvalues accumulating at $\mu^{+}$depends on the behaviour of $\nu^{*}$ in a left neighbourhood of $\mu^{+}$. This behaviour can be described by means of the limit

$$
\lim _{\lambda \rightarrow\left(\mu^{+}\right)^{-}} \nu^{*}(\lambda),
$$

whose existence is guaranteed from Proposition 3.4; more precisely, when the limit in (3.12) is infinite, then there exists $k_{0} \in \mathbb{Z}$ such that for every $k \in \mathbb{Z}$, $k \geq k_{0}$, there exists $\lambda_{k} \in \Lambda$ for which (3.9) holds true and

$$
\lim _{k \rightarrow+\infty} \lambda_{k}=\mu^{+},
$$

i.e. there is accumulation of eigenvalues at $\mu^{+}$. On the other hand, when the limit in (3.12) is finite, then there exists $M^{+} \in \mathbb{R}$ such that

$$
\nu^{*}\left(\frac{\mu^{+}+\mu^{-}}{2}\right)<\nu^{*}(\lambda)<M^{+}, \quad \forall \lambda \in\left(\frac{\mu^{+}+\mu^{-}}{2}, \mu^{+}\right) ;
$$

this implies that there is at most a finite number of eigenvalues of $A_{0}$ in $\left(\left(\mu^{+}+\mu^{-}\right) / 2, \mu^{+}\right)$, i.e. there is not accumulation of eigenvalues at $\mu^{+}$.

Now, let us observe that the fact that the limit in (3.12) is finite or infinite depends on the analogous limit

$$
\lim _{\lambda \rightarrow\left(\mu^{+}\right)^{-}} \nu(\lambda),
$$

since the function $\nu_{*}$ is bounded in $\Lambda$. We are able to show that the finiteness of the limit in (3.13) depends on the behaviour of (2.1) when $\lambda=\mu^{+}$; to this aim, let us observe that a more careful analysis proves that Proposition 2.11 and Proposition 2.17 hold true also when $\lambda=\mu^{+}$. This implies that we are allowed to consider the solution $\zeta_{1, \lambda}$ of (2.1) with $\lambda=\mu^{+}$satisfying (2.50) and the corresponding angular coordinate $\vartheta\left(\cdot, \mu^{+}\right)$, normalized in such a way that $\vartheta\left(0, \mu^{+}\right) \in(0, \pi)$.

Lemma 3.7. Assume

$$
\lim _{x \rightarrow+\infty} \vartheta\left(x, \mu^{+}\right)=\theta^{+} \in \mathbb{R}
$$

then we have

$$
\lim _{\lambda \rightarrow\left(\mu^{+}\right)^{-}} \nu(\lambda)<+\infty .
$$

Proof. Let us observe that (3.14) implies that there exist $\Phi \in \mathbb{R}$ and $X>0$ such that

$$
\vartheta\left(x, \mu^{+}\right)<\Phi, \quad \forall x \geq X .
$$


Moreover, from the monotonicity of $\vartheta(x, \cdot)$, for every $x \geq X$, we deduce that

$$
\vartheta(x, \lambda) \leq \vartheta\left(x, \mu^{+}\right)<\Phi, \quad \forall \lambda<\mu^{+} .
$$

Therefore, for every $\lambda \in \Lambda$ the function $\vartheta(\cdot, \lambda)$ is bounded from above by $\Phi$ in $[X,+\infty)$, hence we have

$$
\nu(\lambda)=\lim _{x \rightarrow+\infty} \vartheta(x, \lambda) \leq \Phi, \quad \forall \lambda<\mu^{+} .
$$

This is sufficient to conclude that (3.15) holds true.

Lemma 3.8. Assume

$$
\lim _{x \rightarrow+\infty} \vartheta\left(x, \mu^{+}\right)=+\infty
$$

and that there exists $X>0$ such that

$$
p_{11}(x)<\mu^{-}, \quad \forall x \geq X .
$$

Then we have

$$
\lim _{\lambda \rightarrow\left(\mu^{+}\right)^{-}} \nu(\lambda)=+\infty .
$$

Proof. Let us first observe that for every $\lambda \in \mathbb{R}$ the angular function $\vartheta(\cdot, \lambda)$ satisfies the differential equation

$$
\theta^{\prime}=\left(\lambda-p_{11}(x)\right) \cos ^{2} \theta-2 p_{12}(x) \cos \theta \sin \theta+\left(\lambda-p_{22}(x)\right) \sin ^{2} \theta .
$$

From (3.19) and (3.17) we deduce that

$$
\forall x \geq X, \lambda>\mu^{-}: \quad \vartheta(x, \lambda)=0(\bmod \pi) \quad \Rightarrow \quad \vartheta^{\prime}(x, \lambda)>0 ;
$$

hence, if there exist $k \in \mathbb{Z}$ and $x_{k} \geq X$ such that

$$
\vartheta\left(x_{k}, \lambda\right)>k \pi
$$

for some $\lambda>\mu^{-}$, then we can conclude that

$$
\vartheta(x, \lambda)>k \pi, \quad \forall x \geq x_{k} .
$$

Now, let us note that (3.16) implies that for every $M>0$ there exists $x_{M} \geq X$ such that

$$
\vartheta\left(x, \mu^{+}\right)>M+2+\pi, \quad \forall x \geq x_{M}
$$

and let us fix $X^{+} \geq x_{M}$; the continuity of $\vartheta\left(X^{+}, \cdot\right)$ ensures that there exists $\lambda_{M}<\mu^{+}$such that

$$
\vartheta\left(X^{+}, \lambda\right)>M+1+\pi, \quad \forall \lambda \in\left(\lambda_{M}, \mu^{+}\right) .
$$

According to the above remark, this implies that

$$
\vartheta(x, \lambda)>M+1, \quad \forall x \geq X^{+}, \lambda \in\left(\lambda_{M}, \mu^{+}\right)
$$

and then

$$
\nu(\lambda)=\lim _{x \rightarrow+\infty} \vartheta(x, \lambda)>M, \quad \forall \lambda \in\left(\lambda_{M}, \mu^{+}\right) .
$$

Therefore, for every $M>0$ there exists $\lambda_{M}<\mu^{+}$such that (3.20) holds, i.e.

$$
\lim _{\lambda \rightarrow\left(\mu^{+}\right)^{-}} \nu(\lambda)=+\infty
$$


The question of the existence of eigenvalues can be dealt, arguing as in the proof of Proposition 3.18 in [4], as follows.

Proposition 3.9. Assume that $P$ has the form (2.8), where $\mu_{a} \in \mathbb{R}, k \in \mathbf{Z} \backslash\{0\}$ and $V \in C^{1}(0,+\infty)$ is a strictly increasing negative potential satisfying (2.9), with $\gamma_{\infty}<0$ and $\alpha_{\infty} \in(0,1]$, and $(2.10)$.

Then, the selfadjoint extension $A_{0}$ of the corresponding operator $\tau$ has a sequence of eigenvalues in $(-1,1)$ accumulating at $\lambda=1$.

Proof. We follow the same argument of [4, Prop. 3.15]. We first observe that the differential equation satisfied by $\vartheta(\cdot, 1)$ is

$$
\vartheta^{\prime}(x, 1)=1-\left\langle Q_{P(x)}[\cos \vartheta, \sin \vartheta],[\cos \vartheta, \sin \vartheta]\right\rangle,
$$

where $Q_{P(x)}$ denotes the quadratic form associated to the matrix $P(x)$. By computing the eigenvalues of $P(x)$, we can prove that

$$
\vartheta^{\prime}(x, 1) \geq 1-V(x)-\sqrt{1+\left(\frac{k}{x}+\mu_{a} V^{\prime}(x)\right)^{2}}, \quad \forall x \geq 1 .
$$

From assumption (2.9) we infer that

$$
1-V(x)-\sqrt{1+\left(\frac{k}{x}+\mu_{a} V^{\prime}(x)\right)}=-\frac{\gamma_{\infty}}{x^{\alpha}}+o\left(\frac{1}{x^{\alpha}}\right), \quad x \rightarrow+\infty
$$

this is sufficient to conclude that

$$
\lim _{x \rightarrow+\infty} \vartheta(x, 1)=+\infty .
$$

The result then follows from the application of Proposition 3.8.

A similar result (under more restrictive conditions on $\alpha$ ) has been obtained by Schmid and Tretter [18]; however, in [18] no information on the nodal properties of the eigenfunctions is provided.

\section{The nonlinear eigenvalue problem}

\subsection{A bifurcation result}

In this section we are interested in proving a global bifurcation result for a nonlinear equation of the form

$$
J z^{\prime}+P(x) z=\lambda z+S(x, z) z, \lambda \in \mathbb{R}, x>0, z \in \mathbb{R}^{2},
$$

where $P \in \mathcal{P}_{\mu}$ and $S \in C\left((0,+\infty) \times \mathbb{R}^{2}, M_{S}^{2}\right)$. We denote by $\mathcal{S}$ the set of continuous functions $S:(0,+\infty) \times \mathbb{R}^{2} \longrightarrow M_{S}^{2}$ satisfying the conditions

$\left(\mathcal{S}_{1}\right)$ there exist $\alpha \in L^{\infty}(0,+\infty), \eta_{i j} \in C\left(\mathbb{R}^{2}\right)$ such that $\eta_{i j}(0)=0, i, j=1,2$, and

$$
\left|S_{i, j}(x, z)\right| \leq \alpha(x) \eta_{i j}(z), \quad \forall x>0, \quad z \in \mathbb{R}^{2}, i, j=1,2 ;
$$

$\left(\mathcal{S}_{2}\right)$ for every compact $K \subset \mathbb{R}^{2}$ there exists $A_{K}>0$ such that

$$
\left\|S(x, z)-S\left(x, z^{\prime}\right)\right\| \leq A_{K}\left\|z-z^{\prime}\right\|, \quad \forall x>0, \quad z, z^{\prime} \in K .
$$


Let $\Sigma$ denote the set of nontrivial solutions of (4.1) in $D_{0} \times \Lambda$ and let $\Sigma^{\prime}=\Sigma \cup\left\{(0, \lambda) \in D_{0} \times \Lambda: \lambda\right.$ is an eigenvalue of $\left.A_{0}\right\}$, where $D_{0}$ and $A_{0}$ are as in Sect. 3. We denote by $\|\cdot\|_{0}$ the graph norm induced on $D_{0}$ by $A_{0}$, defined as

$$
\|z\|_{0}^{2}=\|z\|_{L^{2}(0,+\infty)}^{2}+\|\tau z\|_{L^{2}(0,+\infty)}^{2}, \quad \forall z \in D_{0} .
$$

Let $M$ denote the Nemitskii operator associated to $S$, given by

$$
M(z)(x)=S(x, z(x)) z(x), \quad \forall x>0,
$$

for every $z \in D_{0}$. We can show the validity of the following:

Proposition 4.1. Assume that $S \in \mathcal{S}$ and that

$$
\lim _{x \rightarrow+\infty} \alpha(x)=0 \text {, }
$$

where $\alpha$ is given in (4.2). Then $M: D_{0} \longrightarrow L^{2}(0,+\infty)$ is a continuous compact map and satisfies

$$
M(z)=o\left(\|z\|_{0}\right), \quad z \rightarrow 0 .
$$

The proof of Proposition 4.1 is based on the application of the following lemma:

Lemma 4.2. Assume that $z_{0}, f_{0} \in L^{2}(0,1)$ satisfy

$$
\tau z_{0}=f_{0}
$$

and let $\left\{z_{n}\right\} \subset L^{2}(0,1)$ be a sequence such that

$$
\tau z_{n}=f_{n}
$$

for some $f_{n} \in L^{2}(0,1)$. If $z_{n} \rightarrow z_{0}$ and $f_{n} \rightarrow f_{0}$ weakly in $L^{2}(0,1)$, then $M z_{n} \rightarrow M z_{0}$ strongly in $L^{2}(0,1)$.

Proof. Let us apply Theorem 2.15 to the functions $z_{0}$ and $z_{n}$, for every $n \in \mathbb{N}$ : we have

where

$$
\begin{aligned}
& z_{0}(x)=\nu_{0}(x)+w_{0}(x), \\
& z_{n}(x)=\nu_{n}(x)+w_{n}(x), \quad \forall x \in(0,1),
\end{aligned}
$$

$$
\begin{aligned}
& \nu_{n}(x)=c_{n} \zeta_{1}(x), \nu_{0}(x)=c_{0} \zeta_{1}(x) \\
& w_{n}(x)=\int_{0}^{1} G(x, \xi) f_{n}(\xi) d \xi, w_{0}(x)=\int_{0}^{1} G(x, \xi) f_{0}(\xi) d \xi, \quad \forall x \in(0,1) .
\end{aligned}
$$

Since $G \in L^{\infty}((0,1) \times(0,1))$, we deduce that

$$
w_{n}(x) \rightarrow w_{0}(x), \quad \forall x \in(0,1)
$$

by the weak convergence of $f_{n}$. Moreover, the estimate

$$
\left\|w_{n}(x)-w_{0}(x)\right\| \leq\|G\|_{L^{\infty}\left((0,1)^{2}\right)}\left\|f_{n}-f_{0}\right\|_{L^{2}(0,1)}, \quad \forall x \in(0,1),
$$

holds true; the convergence $f_{n} \rightarrow f_{0}$ in $L^{2}(0,1)$ implies that the sequence $\left\{f_{n}\right\}$ is bounded in $L^{2}(0,1)$ and (4.7)-(4.8) ensure then that $w_{n} \rightarrow w_{0}$ in $L^{2}(0,1)$ by the dominated convergence theorem. This condition, together with 
the assumption $z_{n} \rightarrow z_{0}$ in $L^{2}(0,1)$, implies that $\nu_{n} \rightarrow \nu_{0}$ in $L^{2}(0,1)$. Hence, we obtain that $c_{n} \rightarrow c_{0}$, for $n \rightarrow+\infty$, and

$$
\nu_{n} \rightarrow \nu_{0} \quad \text { in } L^{\infty}(0,1) \text { and in } L^{2}(0,1) .
$$

From (4.7)-(4.9) we have

$$
z_{n}(x) \rightarrow z_{0}(x), \quad \forall x \in(0,1) .
$$

On the other hand, from (4.8) and the boundedness of $\left\{f_{n}\right\}$ in $L^{2}(0,1)$ we deduce also that $\left\{w_{n}\right\}$ is bounded in $L^{\infty}(0,1)$; as a consequence, using (4.9), we get that $\left\{z_{n}\right\}$ is bounded in $L^{\infty}(0,1)$. Using assumption $\left(\mathcal{S}_{2}\right)$, from this fact we infer that there exists $C_{1}>0$ such that

$$
\left\|S\left(x, z_{n}(x)\right)-S\left(x, z_{0}(x)\right)\right\| \leq C_{1}\left\|z_{n}(x)-z_{0}(x)\right\|, \quad \forall x \in(0,1) ;
$$

equations (4.10)-(4.11) guarantee that

$$
S\left(x, z_{n}(x)\right) \rightarrow S\left(x, z_{0}(x)\right) \quad \forall x \in(0,1) .
$$

Finally, from (4.11) and the boundedness of $\left\{z_{n}\right\}$ in $L^{\infty}(0,1)$ we also deduce that there exists $C_{2}>0$ such that

$$
\left\|S\left(x, z_{n}(x)\right) z_{n}(x)-S\left(x, z_{0}(x)\right) z_{0}(x)\right\| \leq C_{2}, \quad \forall x \in(0,1), \quad \forall n \geq 1 ;
$$

an application of the Lebesgue convergence Theorem gives

$$
\int_{0}^{1}\left\|S\left(x, z_{n}(x)\right) z_{n}(x)-S\left(x, z_{0}(x)\right) z_{0}(x)\right\|^{2} d x \rightarrow 0, \quad n \rightarrow+\infty,
$$

i.e. $M z_{n} \rightarrow M z$ in $L^{2}(0,1)$.

Proof of Proposition 4.1. First of all, let us observe that it is sufficient to prove the result when $x \in(0,1)$. Indeed, the fact that $P \in L^{\infty}(1,+\infty)$ implies that the graph norm $\|\cdot\|_{0}$, when applied to functions defined on $[1,+\infty)$, is equivalent to the $H^{1}(1,+\infty)$ norm; hence, when $x \in[1,+\infty)$ we can apply $[4$, Prop. 4.3].

1. We first show that $M z \in L^{2}(0,+\infty)$ when $z \in D_{0}$; from Proposition 3.1 we deduce that $z \in L^{\infty}(0,1)$. Therefore there exists $C_{z}>0$ such that

$$
|S(x, z(x))| \leq C_{z}, \quad \forall x \in(0,1) .
$$

As a consequence we obtain $M z \in L^{\infty}(0,1) \subset L^{2}(0,1)$.

2. Let us fix $z_{0} \in D_{0}$ and let $z_{n} \in D_{0}$ such that $z_{n} \rightarrow z_{0}$ when $n \rightarrow+\infty$; this implies that

$$
z_{n} \rightarrow z_{0} \text { in } L^{2}(0,1), \quad \tau z_{n} \rightarrow \tau z_{0} \text { in } L^{2}(0,1)
$$

We can then apply Lemma 4.2 and obtain that $M z_{n} \rightarrow M z_{0}$ in $L^{2}(0,1)$.

3. As far as the compactness of $M$ is concerned, let $\left\{z_{n}\right\} \subset L^{2}(0,1)$ be such that

$$
\left\|z_{n}\right\|_{0} \leq K
$$

for some $K>0$. This implies that, up to a subsequence, we have

$$
z_{n} \rightarrow z_{0} \text { in } L^{2}(0,1), \quad \tau z_{n} \rightarrow \tau z_{0} \quad \text { in } L^{2}(0,1) .
$$

Hence, according to Lemma 4.2 , we conclude that $M z_{n} \rightarrow M z_{0}$ in $L^{2}(0,1)$. 
4. Finally, let us prove (4.5). We have

$$
\|M z\|_{L^{2}(0,1)}^{2}=\int_{0}^{1}\|M z(x)\|^{2} d z \leq \int_{0}^{1}\|S(x, z(x))\|^{2}\|z(x)\|^{2} d x, \quad \forall z \in D_{0} .
$$

Assume now that $z \rightarrow 0$ in $D_{0}$; this implies that $z \rightarrow 0$ and $\tau z \rightarrow 0$ in $L^{2}(0,1)$; arguing as in the proof of Lemma 4.2 , we deduce that $z \rightarrow 0$ in $L^{\infty}(0,1)$. Therefore, assumption $\mathcal{S}_{2}$ implies that there exists $C>0$ such that

$$
\|S(x, z(x))\| \leq C\|z(x)\| \leq C\|z\|_{L^{\infty}(0,1)}, \quad \forall x \in(0,1) .
$$

From (4.16) and (4.17) we deduce that

$$
\|M z\|_{L^{2}(0,1)} \leq C\|z\|_{L^{\infty}(0,1)}\|z\|_{L^{2}(0,1)} \leq C\|z\|_{L^{\infty}(0,1)}\|z\|_{0},
$$

which implies that $M z=o\left(\|z\|_{0}\right)$ as $\|z\|_{0} \rightarrow 0$.

Now, let us observe that, in view of the results on $A_{0}$ given in Sect. 3 and of Proposition 4.1, it is possible to write (4.1) as an abstract equation of the form

$$
A_{0} u+M(u)=\lambda u,(u, \lambda) \in D_{0} \times \mathbb{R},
$$

where $A_{0}: D_{0} \subset L^{2}(0,+\infty) \rightarrow L^{2}(0,+\infty)$ is an unbounded self-adjoint operator such that

$$
\sigma_{e s s}\left(A_{0}\right)=\left(-\infty, \mu^{-}\right] \cup\left[\mu^{+},+\infty\right)
$$

and $M: D_{0} \times \mathbb{R} \longrightarrow L^{2}(0,+\infty)$ is a continuous and compact map such that

$$
M(u)=o(\|u\|), \quad u \rightarrow 0 .
$$

From an application of a global bifurcation result (see [22, Th. 1.2], [4, Th. $4.1])$ to (4.18) we then obtain the following main result:

Theorem 4.3. Assume that $P \in \mathcal{P}_{\mu}, S \in \mathcal{S}$ and that (4.4) holds true. Then, for every eigenvalue $\gamma \in \Lambda$ of $A_{0}$ there exists a continuum $C_{\gamma}$ of nontrivial solutions of (4.1) in $D_{0} \times \mathbb{R}$ bifurcating from $(0, \gamma)$ and such that one of the following conditions holds true:

(1) $C_{\gamma}$ is unbounded in $D_{0} \times \Lambda$;

(2) $\sup \left\{\lambda:(u, \lambda) \in C_{\gamma}\right\} \geq \mu^{+}$or $\inf \left\{\lambda:(u, \lambda) \in C_{\gamma}\right\} \leq \mu^{-}$;

(3) $C_{\gamma}$ contains $\left(0, \gamma^{\prime}\right) \in \Sigma^{\prime}$, with $\gamma^{\prime} \neq \gamma$.

Now, let us observe that a more precise description of the bifurcating branch, eventually leading to exclude condition (3), can be obtained when there exists a continuous functional $i: \Sigma^{\prime} \rightarrow \mathbb{Z}$ (cf. [4, Th. 4.2]). In order to define such a functional, we first define the rotation number of solutions to $(4.1)$ by means of a linearization procedure; to this aim for every solution $(w, \mu)$ of $(4.1)$ we consider the linear equation

$$
J z^{\prime}+P(x) z=\mu z+S(x, w(x)) z,
$$

which obviously reduces to

$$
J z^{\prime}+P(x) z=\mu z
$$


when $w=0$. It is clear that $w$ is a solution of (4.20); let us denote by $P_{w}$ the matrix defined by

$$
P_{w}(x)=P(x)-S(x, w(x)), \quad \forall x>0 .
$$

We can prove the following result:

Lemma 4.4. For every $(w, \mu) \in \Sigma$ we have $P_{w} \in \mathcal{P}_{\mu}$.

Proof. Let us first observe that $w \in D_{0}$ implies that $w \in H^{1}(1,+\infty)$ and $w \in L^{\infty}(0,+\infty)$ (cf. Proposition 3.1). In particular we have

$$
\lim _{x \rightarrow+\infty} w(x)=0
$$

and there exists a compact set $K_{w} \subset \mathbb{R}^{2}$ such that

$$
w(x) \in K_{w}, \quad \forall x>0 .
$$

Using (2.4), assumption $\left(\mathcal{S}_{1}\right)$ and the fact that $w \in L^{\infty}(0,1)$, we obtain that

$$
\lim _{x \rightarrow 0^{+}} x^{\beta} P_{w}(x)=\lim _{x \rightarrow 0^{+}}\left(x^{\beta} P(x)-x^{\beta} S(x, w(x))\right)=P^{*} ;
$$

therefore $P_{w}$ satisfies (2.4). Moreover, we have

$$
R_{0, w}(x)=x^{\beta} P_{w}(x)-P^{*}=R_{0}(x)-x^{\beta} S(x, w(x)), \quad \forall x>0 .
$$

Using again $\left(\mathcal{S}_{1}\right)$, we plainly deduce that there exists $\eta \in C\left(\mathbb{R}^{2}, \mathbb{R}^{+}\right)$such that

$$
\int_{0}^{1} \frac{1}{x^{\beta}}\left\|x^{\beta} S(x, w(x))\right\|^{q_{0}} d x=\int_{0}^{1} x^{\beta\left(q_{0}-1\right)} \alpha(x) \eta(w(x)) d x<+\infty,
$$

since $q_{0} \geq 1$ and $\alpha, w \in L^{\infty}(0,1)$. From (2.5), (4.23) and (4.24) we can conclude that $R_{0, w}$ satisfies $(2.5)$.

Now, we pass to the proof of the validity of $\left(\mathcal{P}_{1}\right)$. Using $(2.2)$, assumption $\left(\mathcal{S}_{1}\right)$ and $(4.22)$, we infer that

$$
\lim _{x \rightarrow+\infty} P_{w}(x)=\lim _{x \rightarrow+\infty}(P(x)-S(x, w(x)))=P_{\infty} ;
$$

hence $P_{w}$ satisfies $(2.2)$.

Moreover, we have

$$
R_{\infty, w}(x)=P_{w}(x)-P_{\infty}=R_{\infty}(x)-S(x, w(x)), \quad \forall x>0 .
$$

From assumption $\left(\mathcal{S}_{2}\right)$, with $K=K_{w}$ and $z^{\prime}=0$, we obtain

$$
\int_{1}^{+\infty}\|S(x, w(x))\|^{2} d x \leq A_{K_{w}}^{2} \int_{1}^{+\infty}\|w(x)\|^{2} d x<+\infty .
$$

When $q_{\infty} \geq 2$ this allows to conclude that $R_{\infty, w}$ satisfies $(2.3)$, since

$$
\begin{aligned}
& \int_{1}^{+\infty}\|S(x, w(x))\|^{q_{\infty}} d x=\int_{1}^{+\infty}\|S(x, w(x))\|^{q_{\infty}-2}\|S(x, w(x))\|^{2} d x \\
& \quad \leq C_{w} \int_{1}^{+\infty}\|S(x, w(x))\|^{2} d x<+\infty .
\end{aligned}
$$


Finally, also when $q_{\infty}<2$ it is possible to show that $R_{\infty, w}$ satisfies $(2.3)$ with the same $q_{\infty}$ of $R_{\infty}$; indeed, at this point we can say that $w \in H^{1}(1,+\infty)$ is a nontrivial solution of the linear equation

$$
J z^{\prime}+P_{w}(x) z=\mu z,
$$

where $P_{w} \in \mathcal{P}_{\mu}$ and $\mu \in \Lambda$. Therefore Proposition 2.4 applies and we deduce that $w$ satisfies the first condition in $(2.26)$. As a consequence, $w \in L^{q_{\infty}}(1,+\infty)$ and we are able to repeat (4.26) with the exponent $q_{\infty}$ instead of 2 .

As a consequence of Lemma 4.4, the results of Sect. 2 apply to (4.20); in particular, when $w \neq 0$ we can consider the number rot $(w)$ defined in (2.65).

Definition 4.5. Assume that $P \in \mathcal{P}_{\mu}$ and $S \in \mathcal{S}$ and let $(w, \mu)$ be a solution of (4.1).

If $(w, \mu) \neq(0, \mu)$, then the rotation number $j(w, \mu)$ of $(w, \mu)$ is defined by

$$
j(w, \mu)=\operatorname{rot}(w) .
$$

If $(w, \mu)=(0, \mu)$ and the linear problem (4.21) has a nontrivial solution $z_{\mu}$ belonging to $H^{1}(0,+\infty)$, then the rotation number $j(w, \mu)$ of $(w, \mu)$ is defined by

$$
j(w, \mu)=\operatorname{rot}\left(z_{\mu}\right) .
$$

By means of Definition 4.5 we have defined $j: \Sigma^{\prime} \rightarrow \mathbb{R}$; this functional will be used in order to construct a continuous discrete functional whose values are preserved in the bifurcating branches $C_{\gamma}$ of solutions of (4.1). It is important now to observe that every branch $C_{\gamma}$ satisfies

$$
C_{\gamma} \subset H_{0}^{1}(0,+\infty) \times \mathbb{R}
$$

indeed, this is a consequence that $(z, \lambda) \in C_{\gamma}$ is a solution of the linear equation

$$
J z^{\prime}+P_{z}(x) z=\lambda z
$$

such that $z \in D_{0}$. According to Remark 3.2 this implies that $z \in H_{0}^{1}(0,+\infty)$.

Hence, it is sufficient to study the continuity properties of $j$ with respect to the $H_{0}^{1}(0,+\infty)$-norm, denoted by $\||\cdot|\|$.

Proposition 4.6. The function $j: \Sigma^{\prime} \rightarrow \mathbb{R}$ is continuous.

Proof. We prove the continuity of $j$ at every point $(w, \mu) \in \Sigma \cap H_{0}^{1}(0,+\infty)$. In a very similar way it is possible to show that $j$ is also continuous at every point $(0, \lambda)$, with $\lambda$ eigenvalue of $A_{0}$.

Let us fix $(w, \mu) \in \Sigma \cap H_{0}^{1}(0,+\infty)$ and let $\epsilon>0$ small enough; consider then the numbers $\delta, \delta_{0}, x_{\infty}$ and $x_{0}$ given in Proposition 2.21 and Proposition 2.22 (with $\tilde{\lambda}=\mu$ and $\left.\tilde{P}=P_{w}\right)$ and let $\delta_{1}=\min \left(\delta, \delta_{0}\right)$.

Using assumption $\left(\mathcal{S}_{1}\right)$ and the continuous embedding $H_{0}^{1}(0,+\infty) \subset$ $L^{\infty}(0,+\infty)$, it is possible to show that there exist $\delta_{2}>0$ such that

$$
\left\|P_{z}-P_{w}\right\|_{L^{\infty}(0,+\infty)}<\delta_{1}
$$

if ||$|z-w \|| \mid<\delta_{2}$. 
Hence, from Proposition 2.21 and Proposition 2.22 we deduce that for every $(z, \lambda) \in \Sigma \cap H_{0}^{1}(0,+\infty)$ with $|\lambda-\mu|<\delta_{2}$ and $\left\||z-w \||<\delta_{2}\right.$ we have

$$
\begin{aligned}
& \left|\theta_{z}(x, \lambda)-\theta_{z}(+\infty, \lambda)\right|<\epsilon, \quad \forall x \geq x_{\infty} \\
& \left|\theta_{z}(x, \lambda)-\theta_{z}(0)\right|<\epsilon, \quad \forall x \in\left(0, x_{0}\right] .
\end{aligned}
$$

Now, let us observe that we have

$$
\begin{aligned}
j(z, \lambda)-j(w, \mu) & =\frac{\theta_{z}(+\infty, \lambda)-\theta_{z}(0)}{\pi}-\frac{\theta_{w}(+\infty, \mu)-\theta_{w}(0)}{\pi} \\
& =\frac{\theta_{z}(+\infty, \lambda)-\theta_{w}(\infty, \mu)}{\pi},
\end{aligned}
$$

since $\theta_{z}(0)=\theta_{w}(0)$. Therefore, the result follows from the same argument used in the proof of Proposition 3.5.

Before defining the functional $i$ a remark is in order; we recall that if $(w, \lambda) \in \Sigma \cap H_{0}^{1}(0,+\infty)$ then

$$
\theta_{w}(0)=\arctan \frac{w_{1,2}^{*}}{w_{1,1}^{*}} \in(0, \pi) \bmod \pi
$$

and

$$
\theta_{w}(+\infty, \lambda)=\pi-\arctan \sqrt{\frac{\lambda-\mu^{-}}{\mu^{+}-\lambda}} \in\left(\frac{\pi}{2}, \pi\right) \bmod \pi .
$$

As a consequence, when

$$
\arctan \frac{w_{1,2}^{*}}{w_{1,1}^{*}} \in\left(0, \frac{\pi}{2}\right)
$$

we have

$$
j(w, \lambda) \notin \mathbb{Z}, \quad \forall(w, \lambda) \in \Sigma \cap H_{0}^{1}(0,+\infty) .
$$

On the other hand, if

$$
\arctan \frac{w_{1,2}^{*}}{w_{1,1}^{*}} \in\left(\frac{\pi}{2}, \pi\right)
$$

we have

$$
j(w, \lambda)+\frac{1}{2} \notin \mathbb{Z}, \quad \forall(w, \lambda) \in \Sigma \cap H_{0}^{1}(0,+\infty) .
$$

This suggests to define $i: \Sigma^{\prime} \rightarrow \mathbb{Z}$ as

$$
i(w, \lambda)=[j(w, \lambda)], \quad \forall(w, \lambda) \in \Sigma^{\prime},
$$

if (4.31) holds true, and

$$
i(w, \lambda)=\left[j(w, \lambda)+\frac{1}{2}\right], \quad \forall(w, \lambda) \in \Sigma^{\prime},
$$

if (4.32) holds true (recall also Proposition 3.6). Let us observe that Proposition 3.6 also implies that

$$
i\left(z_{\gamma}, 0\right) \neq i\left(z_{\gamma^{\prime}}, 0\right)
$$


for every $\gamma \neq \gamma^{\prime} \in \Lambda$ eigenvalues of $A_{0}$ (with associated eigenfunctions $z_{\gamma}$ and $z_{\gamma^{\prime}}$, respectively).

From Proposition 4.6 and the definition of $i$ we obtain the following result:

Proposition 4.7. The function $i: \Sigma^{\prime} \rightarrow \mathbb{R}$ is continuous.

As a consequence, using Proposition 4.7 and (4.35), from Theorem 4.3 we deduce the final result:

Theorem 4.8. Assume that $P \in \mathcal{P}_{\mu}, S \in \mathcal{S}$ and that (4.4) hold true. Then, for every eigenvalue $\gamma \in \Lambda$ of $A_{0}$ there exists a continuum $C_{\gamma}$ of nontrivial solutions of (4.1) in $D_{0} \times \mathbb{R}$ bifurcating from $(0, \gamma)$ and such that one of the conditions (1)-(2) of Theorem 4.3 holds true and

$$
i(w, \lambda)=i\left(z_{\gamma}, 0\right), \quad \forall(w, \lambda) \in C_{\gamma},
$$

where $z_{\gamma}$ is the eigenfunction of $A_{0}$ associated to $\gamma$.

\subsection{Application to the Dirac equation}

Let us consider the partial differential equation

$$
\begin{gathered}
i \sum_{j=1}^{3} \alpha_{j} \frac{\partial \psi}{\partial x_{j}}-\beta \psi-V(\|x\|) \psi+i a \sum_{j=1}^{3} \alpha_{j} \frac{\partial V(\|x\|)}{\partial x_{j}} \psi \\
\quad=\lambda \psi+\gamma(\|x\|) F(\langle\beta \psi, \psi\rangle) \beta \psi, \quad x \in \mathbb{R}^{3}, a \in \mathbb{R},
\end{gathered}
$$

where $\psi: \mathbb{R}^{3} \rightarrow \mathbb{C}^{4}, V \in C((0,+\infty), \mathbb{R})$ satisfies (2.9)-(2.10)-(2.11)-(2.12), $\gamma \in C((0,+\infty), \mathbb{R})$ fulfills

$$
\lim _{r \rightarrow 0^{+}} r^{2} \gamma(r) \in \mathbb{R}, \quad r^{2} \gamma(r)=o(1), r \rightarrow+\infty,
$$

$F \in C(\mathbb{R}, \mathbb{R}),\langle\cdot, \cdot\rangle$ denotes the scalar product in $\mathbb{C}^{4}$ and $\alpha_{j}(j=1,2,3)$ and $\beta$ are the $4 \times 4$ matrices given by

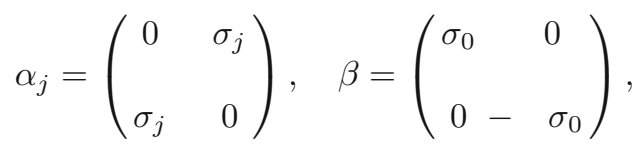

where

$$
\sigma_{0}=\left(\begin{array}{ll}
1 & 0 \\
0 & 1
\end{array}\right), \quad \sigma_{1}=\left(\begin{array}{ll}
0 & 1 \\
1 & 0
\end{array}\right), \quad \sigma_{2}=\left(\begin{array}{cc}
0 & -i \\
i & 0
\end{array}\right), \quad \sigma_{3}=\left(\begin{array}{cc}
1 & 0 \\
0 & -1
\end{array}\right) .
$$

We remark that nonlinearities like the one in (4.37) give rise to the so-called generalized Soler models (see [14]). In fact, Soler [21] formulated a model of extended fermions by introducing a self interaction term which corresponds to the choice $F(s)=s$ in (4.37) (see [17] for a survey on interaction terms which are interesting from a physical point of view).

We denote by $H_{0}$ the (free) Dirac operator defined by

$$
H_{0} \psi=i \sum_{j=1}^{3} \alpha_{j} \frac{\partial \psi}{\partial x_{j}}-\beta \psi, \quad \forall \psi \in H_{0}^{1}\left(\mathbb{R}^{3}\right) \subset L^{2}\left(\mathbb{R}^{3}\right) .
$$


In [23] a decomposition of $H_{0}-V+i a \alpha \cdot \nabla V$ has been performed, using polar coordinates in $\mathbb{R}^{3}$ and the unitary isomorphism

$$
\begin{aligned}
\varphi: & L^{2}\left(\mathbb{R}^{3}\right) \rightarrow L^{2}\left((0,+\infty), d r ; L^{2}\left(S^{2}\right)\right) \\
\psi & \mapsto \tilde{\psi},
\end{aligned}
$$

where $\tilde{\psi}$ is defined by

$$
\tilde{\psi}(r, \theta, \phi)=r \psi(x(r, \theta, \phi)), \quad \forall r>0,(\theta, \phi) \in S^{2} .
$$

In order to describe such a decomposition, for every $l=0,1,2, \ldots$ and $m=$ $-l,-l+1, \ldots, l$ let us denote by $Y_{l}^{m}$ the usual spherical harmonic; moreover, for every $j=1 / 2,3 / 2,5 / 2, \ldots$, let $m_{j}=-j,-j+1, \ldots, j$ and $k_{j}=-(j+$ $1 / 2), j+1 / 2$ and define

$$
\begin{aligned}
& \Psi_{j-1 / 2}^{m_{j}}=\frac{1}{\sqrt{2 j}}\left(\begin{array}{c}
\sqrt{j+m_{j}} Y_{j-1 / 2}^{m_{j}-1 / 2} \\
\sqrt{j-m_{j}} Y_{j-1 / 2}^{m_{j}+1 / 2}
\end{array}\right), \\
& \Psi_{j+1 / 2}^{m_{j}}=\frac{1}{\sqrt{2 j+2}}\left(\begin{array}{c}
\sqrt{j+1-m_{j}} Y_{j+1 / 2}^{m_{j}-1 / 2} \\
-\sqrt{j+1+m_{j}} Y_{j+1 / 2}^{m_{j}+1 / 2}
\end{array}\right)
\end{aligned}
$$

and

$$
\Phi_{m_{j}, \mp(j+1 / 2)}^{+}=\left(\begin{array}{l}
i \Psi_{j \mp 1 / 2}^{m_{j}} \\
0
\end{array}\right), \quad \Phi_{m_{j}, \mp(j+1 / 2)}^{-}=\left(\begin{array}{l}
0 \\
\Psi_{j \pm 1 / 2}^{m_{j}}
\end{array}\right) .
$$

We also set

$$
\mathcal{H}_{m_{j}, k_{j}}=\operatorname{span}\left(\Phi_{m_{j}, k_{j}}^{+}, \Phi_{m_{j}, k_{j}}^{-}\right), \quad \forall j=1 / 2,3 / 2, \ldots
$$

Then, we have the following result:

Theorem 4.9. [23, Th. 4.14] For every $j=1 / 2,3 / 2, \ldots$ the subspace $C_{0}^{\infty}(0,+$ $\infty) \otimes \mathcal{H}_{m_{j}, k_{j}} \subset L^{2}\left((0,+\infty), d r ; L^{2}\left(S^{2}\right)^{4}\right)$ is invariant under the action of $H_{0}-$ $V+i a \alpha \cdot \nabla V$. Moreover, with respect to the basis $\left\{\Phi_{m_{j}, k_{j}}^{+}, \Phi_{m_{j}, k_{j}}^{-}\right\}$of $\mathcal{H}_{m_{j}, k_{j}}$ the restriction of $H_{0}-V+i a \alpha \cdot \nabla V$ to $\mathcal{H}_{m_{j}, k_{j}}$ can be represented by the operator $h_{m_{j}, k_{j}}$ given by

$$
h_{m_{j}, k_{j}}=\left(\begin{array}{cc}
-1-V & \frac{d}{d r}-\frac{k_{j}}{r}+a V^{\prime} \\
-\frac{d}{d r}-\frac{k_{j}}{r}+a V^{\prime} & 1-V
\end{array}\right) .
$$

Moreover, the Dirac operator $H_{0}-V+i a \alpha \cdot \nabla V$ on $C_{0}^{\infty}\left(\mathbb{R}^{3}\right)^{4}$ is unitarily equivalent to the direct sum of the partial waves operators $h_{m_{j}, k_{j}}$, i.e.

$$
H_{0}-V+i a \alpha \cdot \nabla V \approx \bigoplus_{j=1 / 2,3 / 2, \ldots}^{+\infty} \bigoplus_{m_{j}=-j}^{j} \bigoplus_{k_{j}= \pm(j+1 / 2)} h_{m_{j}, k_{j}} .
$$


Remark 4.10. The partial wave subspaces can be considered as a suitable generalization of radial functions adapted to the structure of the nonlinear problem. More precisely, the vectors $\Phi_{m_{j}, k_{j}}^{ \pm}$which are a basis for the partial wave subspace $\mathcal{H}_{m_{j}, k_{j}}$ are the eigenfunctions of the spin orbit operator (cf. ([23]). We also observe that these subspaces are implicitly used in [1,2], where (having in mind the Soler model) the system of ODEs is obtained from the PDE by making the ansatz that solutions should be a linear combination of functions of the form $\Phi_{1 / 2,1}^{+}=\left(\frac{i}{2 \sqrt{\pi}} \cos \theta, \frac{i}{2 \sqrt{\pi}} e^{i \phi} \sin \theta, 0,0\right), \Phi_{1 / 2,1}^{-}=\left(0,0, \frac{1}{2 \sqrt{\pi}}, 0\right)$. On the same lines but in the context of the Schrödinger equation, we refer to [24, Example 1.5].

Let us observe that the operators $\tau_{k_{j}}=h_{m_{j}, k_{j}}, j=1 / 2,3 / 2, \ldots$, are of the form (3.1) with $P=P_{V, k_{j}, a}$ as in (2.8). Therefore, we can apply the theory developed in Sects. 2 and 3; in particular we can consider the selfadjoint realization $A_{0}$ of $\tau_{k_{j}}, j=1 / 2,3 / 2, \ldots$, defined in (3.2). We denote by $A_{k_{j}}$ this operator, by $D_{k_{j}}$ its domain and we define

$$
E_{k_{j}}=\left\{u^{+} \Phi_{m_{j}, k_{j}}^{+}+u^{-} \Phi_{m_{j}, k_{j}}^{-}: u=\left(u^{+}, u^{-}\right) \in D_{k_{j}}\right\} .
$$

From Theorem 4.9 and the definition of $D_{k_{j}}$ we immediately deduce that the image of $E_{k_{j}}$ via the operator $H_{0}-V+i a \alpha \cdot \nabla V$ is contained in $L^{2}((0, \infty)) \otimes$ $\mathcal{H}_{k_{j}, m_{j}}$, for every $j=1 / 2,3 / 2, \ldots$

Now, let us observe that Theorem 4.9 states that the subspaces

$$
C_{0}^{\infty}(0,+\infty) \otimes \mathcal{H}_{m_{j}, k_{j}} \subset L^{2}\left((0,+\infty), d r ; L^{2}\left(S^{2}\right)^{4}\right), \quad j=1 / 2,3 / 2, \ldots,
$$

are preserved by the linear operator in (4.37). It is important to note that in the particular case of $j=1 / 2$ the subspaces $C_{0}^{\infty}(0,+\infty) \otimes \mathcal{H}_{m_{1 / 2}, k_{1 / 2}} \subset$ $L^{2}\left((0,+\infty), d r ; L^{2}\left(S^{2}\right)^{4}\right)$ are invariant also for the nonlinear term $F(\langle\beta \psi, \psi\rangle) \beta \psi$ in (4.37) (cf. [3, Lemma 5.5]), when $F$ is regular.

Indeed, let $u \in L^{2}\left(\mathbb{R}^{3}\right)^{4}$ such that $\varphi(u) \in C_{0}^{\infty}(0,+\infty) \otimes \mathcal{H}_{m_{1 / 2}, k_{1 / 2}}$, where $\varphi$ is defined in (4.40)-(4.41). A simple computation, based on the expressions of the functions $\Phi_{m_{1 / 2}, k_{1 / 2}}^{ \pm}$, shows that, if we have

$$
(\varphi(u))(r, \theta, \phi)=u^{+}(r) \Phi_{m_{1 / 2}, k_{1 / 2}}^{+}(\theta, \phi)+u^{-}(r) \Phi_{m_{1 / 2}, k_{1 / 2}}^{-}(\theta, \phi),
$$

then

$$
\langle\beta u(x), u(x)\rangle=\frac{1}{4 \pi^{2} r^{2}}\left[\left(u^{+}(r)\right)^{2}-\left(u^{-}(r)\right)^{2}\right]
$$

and

$$
\begin{aligned}
F( & \left.\left\langle\beta \frac{(\varphi(u))(r, \theta, \phi)}{r}, \frac{(\varphi(u))(r, \theta, \phi)}{r}\right\rangle\right) \beta[(\varphi(u))(r, \theta, \phi)] \\
& =F\left(\frac{1}{4 \pi^{2} r^{2}}\left(u^{+}(r)\right)^{2}-\left(u^{-}(r)\right)^{2}\right)\left(u^{+}(r) \Phi_{m_{1 / 2}, k_{1 / 2}}^{+}(\theta, \phi)\right. \\
& \left.-u^{-}(r) \Phi_{m_{1 / 2}, k_{1 / 2}}^{-}(\theta, \phi)\right),
\end{aligned}
$$


proving that

$\tilde{\psi} \in C_{0}^{\infty}(0,+\infty) \otimes \mathcal{H}_{m_{1 / 2}, k_{1 / 2}} \Rightarrow F\left(\left\langle\beta \frac{\tilde{\psi}}{r}, \frac{\tilde{\psi}}{r}\right\rangle\right) \beta \tilde{\psi} \in C_{0}^{\infty}(0,+\infty) \otimes \mathcal{H}_{m_{1 / 2}, k_{1 / 2}}$.

Then, with an argument similar to the one developed in the proof of Proposition 4.1, we deduce that

$$
\tilde{\psi} \in E_{k_{1 / 2}} \Rightarrow \gamma(r) F\left(\left\langle\beta \frac{\tilde{\psi}}{r}, \frac{\tilde{\psi}}{r}\right\rangle\right) \beta \tilde{\psi} \in L^{2}(0,+\infty) \otimes \mathcal{H}_{m_{1 / 2}, k_{1 / 2}}
$$

This fact is important to obtain a relation between solutions of (4.37) and solutions of a nonlinear ordinary differential equation of the form (4.1). Indeed, for every function $u \in L^{2}\left(\mathbb{R}^{3}\right)^{4}$ with $\varphi(u) \in E_{k_{1 / 2}}$, let $z=\left(u^{+}, u^{-}\right) \in D_{k_{1 / 2}}$ such that

$$
\varphi(u)=u^{+} \Phi_{m_{1 / 2}, k_{1 / 2}}^{+}+u^{-} \Phi_{m_{1 / 2}, k_{1 / 2}}^{-}
$$

Then, (4.49) implies that $u \in L^{2}\left(\mathbb{R}^{3}\right)^{4}$ with $\varphi(u) \in E_{k_{1 / 2}}$ is a nontrivial solution of (4.37) if and only if $z=\left(u^{+}, u^{-}\right) \in D_{k_{1 / 2}}$ is a nontrivial solution of

$$
\tau_{k_{1 / 2}} z=\lambda z+\gamma(r) F\left(\frac{\left(u^{+}\right)^{2}-\left(u^{-}\right)^{2}}{4 \pi r^{2}}\right)\left[\begin{array}{rr}
1 & 0 \\
0 & -1
\end{array}\right] z, \quad r>0 .
$$

Let us denote $E=\varphi^{-1}\left(E_{k_{1 / 2}}\right)$; in view of the above arguments and choosing

$$
S(r, z)=\gamma(r) F\left(\frac{\left(u^{+}\right)^{2}-\left(u^{-}\right)^{2}}{4 \pi r^{2}}\right)\left[\begin{array}{rr}
1 & 0 \\
0 & -1
\end{array}\right]
$$

from Theorem 4.8 we plainly obtain the following result:

Theorem 4.11. Let us suppose that $V \in C(0,+\infty)$ and $\gamma \in C(0,+\infty)$ satisfy (2.9)-(2.10)-(2.11) and (4.38) and let $F: \mathbb{R}^{2} \rightarrow \mathbb{R}^{2}$ be a locally Lipschitz continuous function such that $|F(s)| \leq C|s|$ for all $s \in \mathbb{R}^{2}$ and some constant $C>0$. Then, for every eigenvalue $\mu \in(-1,1)$ of $A_{k_{1 / 2}}$ there exists a continuum $C_{\mu}$ of nontrivial solutions of (4.37) in $E \times \mathbb{R}$ such that one of the conditions

(1) $C_{\mu}$ is unbounded in $E \times(-1,1)$

(2) $\sup \left\{\lambda:(u, \lambda) \in C_{\mu}\right\} \geq 1$ or $\inf \left\{\lambda:(u, \lambda) \in C_{\mu}\right\} \leq-1$

holds true and

$$
\tilde{i}(w, \lambda)=i\left(z_{\mu}, 0\right), \quad \forall(w, \lambda) \in C_{\mu},
$$

where

$$
\tilde{i}(w, \lambda)=i\left(\left(w^{+}, w^{-}\right), \lambda\right)
$$

and $z_{\mu}$ is the eigenfunction of $A_{k_{1 / 2}}$ associated to $\mu$. 


\section{References}

[1] Balabane, M., Cazenave, T., Douady, A., Merle, F.: Existence of excited states for a nonlinear Dirac field. Commun. Math. Phys. 119, 153-176 (1988)

[2] Balabane, M., Cazenave, T., Vazquez, L.: Existence of standing waves for Dirac fields with singular nonlinearities. Commun. Math. Phys. 133, 53-74 (1990)

[3] Cacciafesta, F.: Global small solutions to the critical radial Dirac equation with potential. Nonlinear Anal. 74, 6060-6073 (2011)

[4] Capietto, A., Dambrosio, W.: Planar Dirac-type systems: the eigenvalue problem and a global bifurcation result. J. Lond. Math. Soc. 81, 477-498 (2010)

[5] Capietto, A., Dambrosio, W., Papini, D.: A global bifurcation result for a second order singular equation. Rend. Istit. Mat. Univ. Trieste. Special Issue in Honour of Prof. F. Zanolin. 44, 173-185 (2012)

[6] Coppel, W.A.: Dichotomies in stability theory. Lectures Notes in Mathematics, vol. 629 (1978)

[7] Dancer, N.: Boundary-value problems for ordinary differential equations on infinite intervals. Proc. Lond. Math. Soc. 30, 76-94 (1975)

[8] Dancer, N.: Boundary-value problems for ordinary differential equations of infinite intervals. II. Q. J. Math. Oxford Ser. 28, 101-115 (1977)

[9] Ding, J., Xu, J., Zhang, F.: Solutions of super-linear Dirac equations with general potentials. Differ. Equ. Dyn. Syst. 17, 235-256 (2009)

[10] Ding, Y., Ruf, B.: Solutions of a nonlinear Dirac equation with external fields. Arch. Rational Mech. Anal. 190, 57-82 (2008)

[11] Dong, Y., Xie, J.: Admissible solutions for Dirac equations with singular and non-monotone nonlinearity. Proc. Edinb. Math. Soc. 54, 363-371 (2011)

[12] Dunford, N., Schwartz, J.: Linear Operators-Part II: spectral theory. Interscience Publishers, New York (1963)

[13] Eastham, M.S.P.: The asymptotic solution of linear differential systems. London Math. Society Monographs New Series (1989)

[14] Esteban, M.J.: An overview on linear and nonlinear Dirac equations. Discrete Contin. Dyn. Syst. 8, 381-397 (2002)

[15] Kalf, H., Schmidt, K.M.: Spectral stability of the Coulomb-Dirac Hamiltonian with anomalous magnetic moment. J. Differ. Equ. 205, 408-423 (2004)

[16] Rabier, P.J., Stuart, C.: Global bifurcation for quasilinear elliptic equations on $\mathbb{R}^{N}$. Math. Z. 237, 85-124 (2001)

[17] Rañada, A.F.: On nonlinear classical Dirac fields and quantum physics. In: Old and New Questions in Physics, Cosmology, Philosophy, and Theoretical Biology, pp. 363-376. Plenum, New York (1983) 
[18] Schmid, H., Tretter, C.: Singular Dirac systems ans Sturm-Liouville problems nonlinear in the spectral parameter. J. Differ. Equ. 181, 511-542 (2002)

[19] Schmid, H., Tretter, C.: Eigenvalue accumulation for Dirac operators with spherically symmetric potential. J. Phys. A. 37, 8657-8674 (2004)

[20] Secchi, S., Stuart, C.: Global bifurcation of homoclinic solutions of Hamiltonian systems. Discrete Contin. Dyn. Syst. 9, 1493-1518 (2003)

[21] Soler, M.: Classical, Stable, Nonlinear Spinor Field with Positive Rest Energy. Phys. Rev. D. 1, 2766-2769 (1970)

[22] Stuart, C.: Global properties of components of solutions of non-linear second order differential equations on the half-line. Ann. Scuola Norm. Sup. Pisa Cl. Sci. 4(2), 265-286 (1975)

[23] Thaller, B.: The Dirac Equation. Text and Monographs in Physics (1992)

[24] Weidmann, J.: Spectral theory of ordinary differential equations. Lectures Notes in Mathematics, vol. 1258 (1987)

Anna Capietto and Walter Dambrosio

Dipartimento di Matematica

Università di Torino

Via Carlo Alberto 10

10123 Turin

Italy

e-mail: walter.dambrosio@unito.it

Anna Capietto

e-mail: anna.capietto@unito.it

Duccio Papini

Dipartimento di Ingegneria dell'Informazione e Scienze Matematiche

Università di Siena

Via Roma 56

53100 Siena

Italy

e-mail: duccio.papini@unisi.it

Received: 25 November 2013.

Accepted: 3 July 2014. 